\title{
Blockade of BFA-mediated apoptosis in macrophages by the HIV-1 Nef protein
}

\author{
W Abbas ${ }^{1}$, KA Khan ${ }^{1}$, A Kumar ${ }^{1}$, MK Tripathy ${ }^{1}$, I Dichamp ${ }^{1}$, M Keita ${ }^{1}$, U Mahlknecht ${ }^{2}$, O Rohr ${ }^{3}$ and G Herbein ${ }^{\star, 1}$
}

HIV-1 Nef protein has key roles at almost all stages of the viral life cycle. We assessed the role of Nef and of the translation elongation factor eEF1A in primary human macrophages. Nuclear retention experiments and inhibition of the exportin-t (Exp-t) pathway suggested that cytoplasmic relocalization of eEF1A, mediated by Exp-t occurs in Nef-treated monocyte-derived macrophages (MDMs). We observed the presence of tRNA in the Nef/eEF1A complexes. Nucleocytoplasmic relocalization of the Nef/eEF1A complexes prevented stress-induced apoptosis of MDMs treated with brefeldin A. Blockade of stress-induced apoptosis of MDMs treated with HIV-1 Nef resulted from enhanced nucleocytoplasmic transport of eEF1A with decreased release of mitochondrial cytochrome $c$, and from increased tRNA binding to cytochrome $c$, ultimately leading to an inhibition of caspase activation. Our results indicate that HIV-1 Nef, through the nucleocytoplasmic relocalization of eEF1A and tRNAs, enhances resistance to stress-induced apoptosis in primary human macrophages.

Cell Death and Disease (2014) 5, e1080; doi:10.1038/cddis.2014.16; published online 20 February 2014

Subject Category: Immunity

The endoplasmic reticulum (ER) is a well-orchestrated protein-folding machine composed of protein chaperones, proteins that catalyze protein folding, and sensors that detect the presence of misfolded or unfolded proteins. The unfolded protein response (UPR) is an intracellular signaling pathway that coordinates the ER protein-folding demand with proteinfolding capacity and is essential to adapt to homeostatic alterations that cause protein misfolding. ${ }^{1}$ Accumulating evidence suggests that protein folding and generation of reactive oxygen species (ROS) as a byproduct of protein oxidation in the ER are closely linked events. It has also become apparent that activation of the UPR on exposure to oxidative stress is an adaptive mechanism to preserve cell function and survival. ${ }^{1}$ Persistent oxidative stress and protein misfolding initiate apoptotic cascades and are now known to have predominant roles in the pathogenesis of multiple human diseases including diabetes, atherosclerosis, and neurodegenerative diseases. ${ }^{2}$

Activated monocytes are a major source of ROS that are essential for bacterial killing. ${ }^{3}$ However, excessive ROS production not appropriately compensated by antioxidant molecules can lead to oxidative stress, which may also have an important role in pathogenesis of HIV infection through various mechanisms. ${ }^{4}$ ROS participate in T-lymphocyte depletion by triggering apoptosis; and monocytes can also induce their own apoptosis by producing ROS. ${ }^{5} \mathrm{HIV}$ infection leads to the production of ROS in infected cells and causes transient downregulation of the antiapoptotic $\mathrm{Bcl}-2$ and thioredoxin (TRX) protein expression in Jurkat and U937 cells, followed by restoration to initial levels. Cells with decreased levels of these proteins are susceptible to apoptosis. ${ }^{4}$ In addition, the upregulation of $\mathrm{Bcl}-2$ expression represses HIV replication; therefore, the state of low level viral replication may be favorable for cell survival, resulting in persistent infection. ${ }^{6}$ In monocytes, from asymptomatic patients, the levels of Bcl-2 and TRX decrease, which is associated with enhanced hydrogen peroxide production, whereas in cells from AIDS patients the levels returned to normal. ${ }^{6}$ Thus, the redox balance affected by the intracellular TRX system seems to be important for cell survival and low viral production, probably leading to chronic persistent infection of HIV. HIV-1Tat has been reported to induce UPR, ${ }^{7}$ indicating that ER stress response could be a critical parameter to control during HIV infection.

Nuclear-cytoplasmic transport of all molecules, such as proteins and RNA species, across the nuclear envelope occur

\footnotetext{
${ }^{1}$ Department of Virology, Pathogens \& Inflammation Laboratory, University of Franche-Comte, EA 4266, SFR FED 4234, CHRU Besancon, Besançon F-25030, France; ${ }^{2}$ University of Heidelberg Medical Center, St. Lukas Klinik Solingen, Solingen D-42697, Germany and ${ }^{3}$ Institut de Parasitologie et Pathologie Tropicale, EA 4438, Strasbourg University, 3 rue Koeberlé, Strasbourg 67000, France

*Corresponding author: G Herbein, UPRES EA 4266 Department of Virology, Pathogens \& Inflammation Laboratory, University of Franche-Comte, CHRU Besancon, 2 place Saint- Jacques, Besançon cedex F-25030, France. Tel: +33 3812188 77; Fax: +33 3816656 95; E-mail: georges.herbein@ univ-fcomte.fr

Keywords: Nef; eEF1A; tRNA; cytochrome $c$; apoptosis; macrophage

Abbreviations: Ab, antibody; AIDS, acquired immune deficiency syndrome; ANOVA, analysis of variance; Apaf-1, apoptotic peptidase-activating factor-1; ASK-1, apoptosis signal-regulating kinase 1; BFA, brefeldin A; DTT, dithiothreitol; EF1A, translation elongation factor 1 alpha; EF1 $\beta$, elongation factor 1 beta; eEF2, eukaryotic translation elongation factor-2; ER, endoplasmic reticulum; EtBr, ethidium bromide; Exp-t, exportin-t; Exp-1, exportin-1; Exp-5, exportin-5; FBS, fetal bovine serum; GST, glutathione $S$ transferase; HIV, human immunodeficiency virus; IPTG, isopropyl $\beta$-D-1-thiogalactopyranoside; LMB, leptomycin B; MDMs, monocyte-derived macrophages; MHC-I, major histocompatibility complex class I; PAGE, polyacrylamide gel electrophoresis; PAK, p21-activated kinase; PBLs, peripheral blood lymphocytes; PBMCs, peripheral blood mononuclear cells; PI3K, phosphatidylinositol-3 kinase; PMSF, phenylmethylsulfonyl fluoride; ROS, reactive oxygen species; rNef, recombinant Nef; RT-PCR, reverse transcription polymerase chain reaction; TBP, TATA-binding protein; TG, thapsigargin; TM, tunicamycin; TNF, tumor necrosis factor; TRX, thioredoxin; TUNEL, terminal deoxynucleotidyl transferase dUTP nick end labeling; UPR, unfolded protein response; WT, wild type Received 11.1.13; revised 14.12.13; accepted 08.1.14; Edited by G Melino
} 
through the channels formed by macromolecular structures known as nuclear pore complexes. The translation elongation factor EF1A has been described as a cytoplasmic component of the nuclear aminoacylation-dependent tRNA export pathway. ${ }^{8}$ eEF1A interacts directly with aminoacylated tRNAs. Exp-t has been reported to have a major role in eEF1Amediated export, though Exp-5, which preferentially exports pre-miRNAs, has been reported to be involved in the nuclearcytoplasmic transport of tRNAs in some cases. ${ }^{9}$ In addition to the conventional role of eEF1A during protein synthesis, namely GTP-dependent binding and transport of aminoacyltRNA to the A site of the ribosome, eEF1A has a role in the cytoplasm. One of the proposed unconventional functions of eEF1A is a role in the regulation of cytoskeletal dynamics. ${ }^{10}$ In addition to binding GDP/GTP, aminoacyl-tRNA, EF1 $\beta$, and the ribosome, eEF1A binds and bundles actin and binds microtubules. Because eEF1A is an abundant protein in most eukaryotic cells and binds to actin filaments with relatively high affinity, it could be a potent regulator of the cytoskeleton. ${ }^{11}$ Previous in vitro studies demonstrated that eEF1A inhibits the rate of actin polymerization and stabilizes actin filaments. $^{12}$

In addition, eEF1A has been reported to have a role in apoptosis or programmed cell death. Early experiments showed that the level of eEF1A expression in cultured mouse fibroblasts correlates with the rate of apoptosis on serum withdrawal, with higher levels of eEF1A expression associated with a faster rate of cell death. ${ }^{13}$ Other studies have indicated that eEF1A prevents cell death; and numerous studies have shown that eEF1A expression increases in tumor cells and tumor tissues parallel with decreased caspase- 3 activation. ${ }^{14,15}$

Nef, a $27-\mathrm{kDa}$ HIV-1 protein, is translated from multiply spliced viral mRNAs early during infection. ${ }^{16}$ Endogenous Nef may have evolved a number of different, independent functional activities to enhance the replication and survival of the virus within infected cells and to facilitate its spread in vivo. ${ }^{17}$ Nef enhances virion infectivity and increases viral replication in primary lymphocytes and macrophages. ${ }^{18}$ The protein can also mediate downregulation of CD4 cell surface expression, a phenomenon important for the release of HIV-1 from the infected cell. ${ }^{17}$ In addition, Nef can downregulate the cell surface expression of major histocompatibility complex class I (MHC-I) molecules, an effect found to protect infected cells from cytotoxic $\mathrm{T}$ cells. ${ }^{19}$ The Nef protein prevents apoptosis of HIV-1-infected T cells through either interference with $\mathrm{Fas} / \mathrm{TNF} \alpha$ receptor death-signaling pathways by inhibiting apoptosis signal-regulating kinase 1 (ASK-1) ${ }^{20}$ or the formation of a complex with both p21-activated kinase (PAK) and phosphatidylinositol-3 kinase (PI3K), which increases phosphorylation and inactivation of the pro-apoptotic Bad protein. $^{21}$ HIV-1 Nef protects human monocyte-derived macrophages (MDMs) from HIV-1-induced apoptosis, and this protection correlates with the hyperphosphorylation and consequent inactivation of Bad. $^{22}$ Nef expression within macrophages has been reported to favor the recruitment of resting $\mathrm{T}$ cells via the secretion of $\mathrm{C}-\mathrm{C}$ chemokines and to subsequently favor their activation, suggesting a role for Nef in lymphocyte recruitment and activation at sites of viral replication. ${ }^{23}$

Our results indicate that HIV-1 Nef associates with eEF1A and that Exp-t contributes to the nuclear-cytoplasmic transport of Nef/eEF1A/tRNA complexes in macrophages. Finally, we observed that cytoplasmic relocalization of the Nef/ eEF1A/tRNA complexes prevents stress-induced apoptosis in macrophages via increased cytoplasmic eEF1A expression, decreased release of mitochondrial cytochrome $c$, and plugging of released cytochrome $c$ by cytoplasmic tRNAs, ultimately resulting in decreased caspase activation.

\section{Results}

Interaction between HIV-1 Nef and eEF1A. As both Nef and eEF1A modulate apoptosis, ${ }^{14,22}$ we assessed whether eEF1A is part of a eEF1A/Nef complex. To test this interaction, we expressed a Nef-GST fusion protein in $E$. coli and tested its ability to interact with eEF1A from U937 cell lysates. The Nef protein bound to eEF1A (Figure 1a). Endogenous eEF1A protein present in the lysates of Vero cells, MRC5 cells, promonocytic U937 cells, primary peripheral blood lymphocytes (PBLs), and primary MDMs co-immunoprecipitated with recombinant Nef (rNef) added to the culture, whereas the isotype control showed no associated eEF1A protein on immunoprecipitation (Figure 1b). The interaction between eEF1A and rNef was detected in both nuclear and cytoplasmic lysates prepared from the cells (Figure 1b).

The interaction between eEF1A and endogenous Nef was also demonstrated by transient transfection of U937 cells with a nef-expressing plasmid (Figure 1c), and in peripheral blood

\footnotetext{
Figure 1 eEF1A interacts with HIV-1 Nef protein in vitro and in vivo. (a) Binding of HIV-1 Nef to eEF1A was measured in GST pull-down assays using U937 cells as a source of lysates. $\beta$-actin detection represents input loading controls of the lysates that were used in binding reactions. Results are representative of three independent experiments. The right panel represents coomassie staining of expressed proteins that were used in the binding reaction of GST pull-down. (b) Cytoplasmic and nuclear extracts from several cell lines (Vero, MRC5, and U937 cells), PBLs, and MDMs treated with rNef (100 ng/ml) for $0.5 \mathrm{~h}$ were immunoprecipitated with an anti-eEF1A antibody, or Nef $(100 \mathrm{ng} / \mathrm{ml})$-treated total cellular lysates were immunoprecipitated with an isotype control antibody. Immunoprecipitated material was analyzed by western blotting with an anti-Nef monoclonal antibody. Recombinant Nef-treated total cell lysates were used as a positive control. Results are representative of three independent experiments. (c) Cellular extracts of U937 cells transfected for $48 \mathrm{~h}$ with a nef-expressing plasmid (pNef) were immunoprecipitated with an anti-eEF1A antibody, anti-Nef antibody, or isotype control antibody. Immunoprecipitated material was analyzed by western blotting with an anti-Nef monoclonal antibody. Results are representative of two independent experiments. (d) Cellular extracts of PBMCs infected in vitro with HIV-1 $1_{89.6}$ or mock infected were immunoprecipitated with an anti-eEF1A antibody or anti-Nef monoclonal antibody. Immunoprecipitated material was analyzed by western blotting with an anti-Nef monoclonal antibody or anti-eEF1A antibody. Results are representative of two independent experiments. (e and f) eEF1A and HIV-1 Nef interact in vitro in a mammalian two-hybrid assay. (e) Schematic representation of expression constructs used in cotransfection experiments in the mammalian two-hybrid model. (f) Mammalian two-hybrid analysis with EEF1A fused to the VP16 activator domain and HIV-1 Nef fused to the GAL4 domain. Luciferase assays were conducted on total extracts from U937 cells transfected with the luciferase expression construct pG5-Luc, pBIND-Nef, pACT-eEF1A, or control plasmids. As a positive control, two plasmids, pACT-MyoD and pBIND-Id, were co-transfected, and co-transfection of empty vectors was used as a negative control. Results represent the mean of three independent experiments. ${ }^{* * *} P<0.001$
} 
a

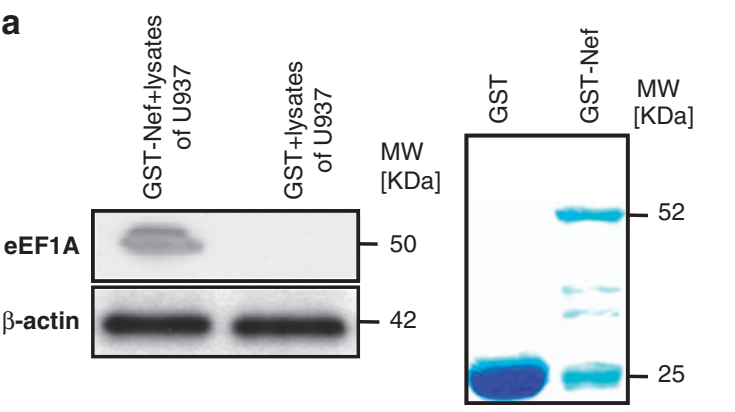

C

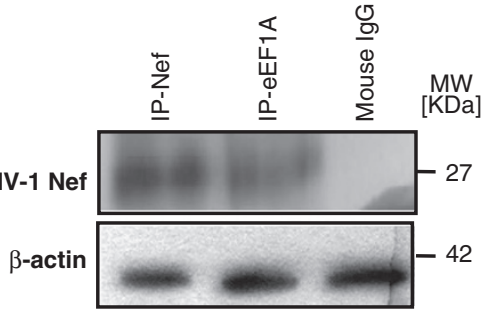

b

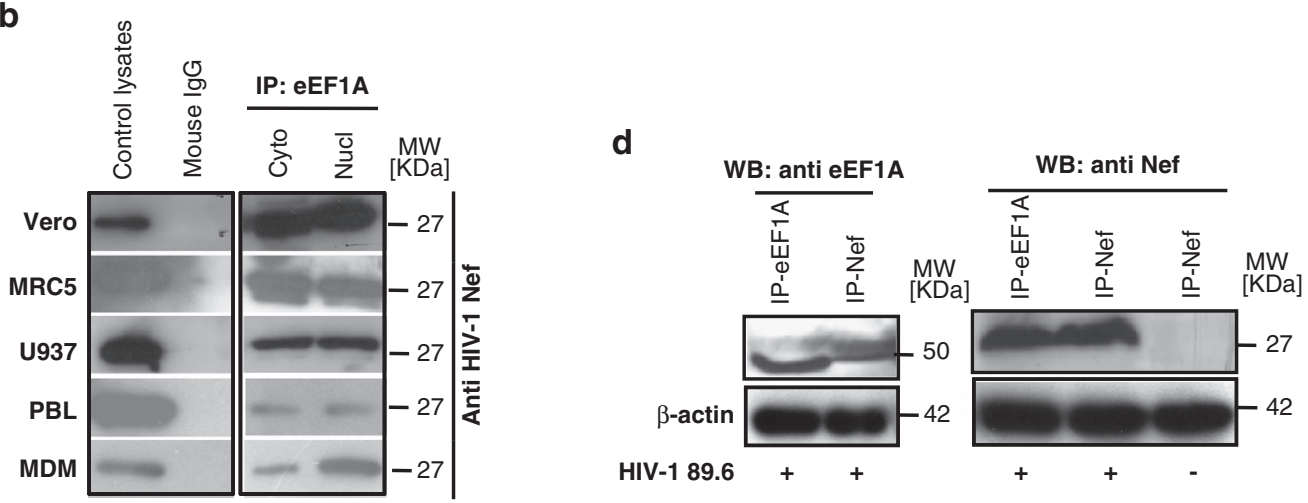

e
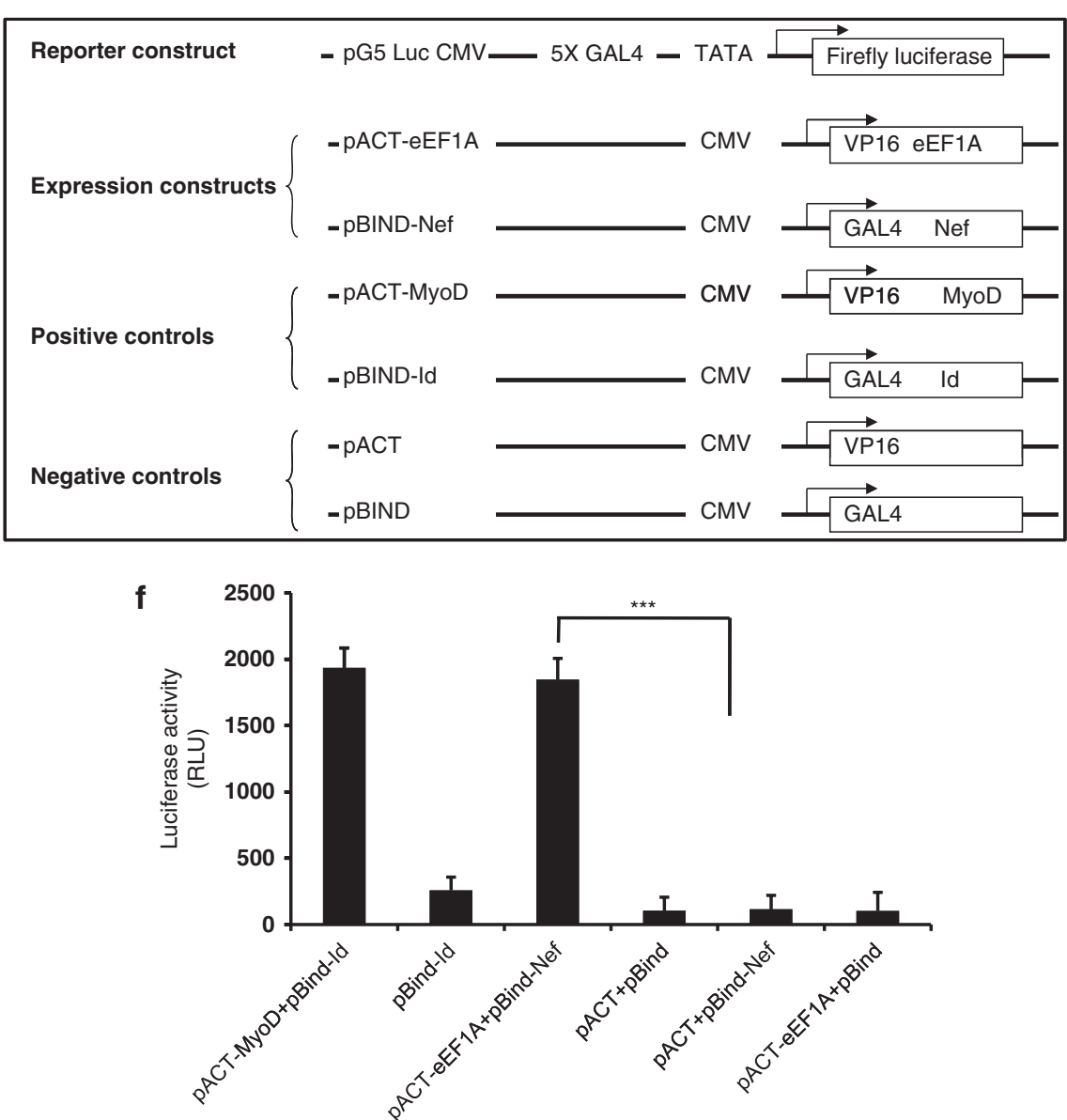
mononuclear cells (PBMCs) infected in vitro with HIV-189.6 (Figure 1d). Thus, eEF1A interacts with the Nef protein, not only in vitro within several cell types treated with rNef but also with the endogenous Nef protein produced within HIV-1infected primary PBMCs. Lysates from MDMs treated with rNef in vitro were immunoprecipitated with antibodies directed against the eukaryotic translation elongation factor-2 (eEF2), and western blot performed with an anti-Nef antibody. The Nef protein did interact with eEF1A, but not with eEF2 (data not shown), indicating that the Nef/eEF1A interaction was specific. We further investigated the interaction between eEF1A and Nef using a mammalian two-hybrid assay. Nef fused to the GAL4 DNA-binding domain (pBIND) was used as the bait vector, and eEF1A fused to the VP16-activation domain ( $p A C T$ ) was used as the prey vector (Figure 1e). The constructs were transfected into mammalian cells along with the pG5-Luc vector, which contains five GAL4-binding sites upstream of a minimal TATA box upstream of the luciferase gene. The interaction between the GAL4-Nef and VP16eEF1A fusion constructs resulted in an 18-fold increase of relative luciferase expression over the negative control (1847 versus 105 relative light units (r.l.u.); Figure 1f). Expression of pGAL4-Nef alone or pVP16- eEF1A alone did not result in increased luciferase activity compared with negative controls (115 r.l.u. and 103 r.l.u., respectively).

\section{Determination of eEF1A and HIV-1 Nef regions involved} in eEF1A-Nef interaction. To determine the region of eEF1A responsible for association with HIV-1 Nef, we used deletion mutants engineered within the cDNA encoding eEF1A. ${ }^{24}$ Lysates from bacteria expressing $\mathrm{N}$-terminal HAtagged eEF1A mutants were used in binding reactions with GST-HIV-1 Nef. The deletion mutants we analyzed are shown in Figure 2a. Three of the deletion mutants were not stably expressed. ${ }^{24}$ Of the deletion mutants that were expressed, only the eEF1A 1-74 mutant was capable of binding Nef (Figure 2a). These results demonstrate that the eEF1A fragment encoding the $\mathrm{N}$-terminal 74 amino acids is sufficient for HIV-1 Nef binding. To determine the region of HIV-1 Nef responsible for binding to eEF1A, two GST-Nef deletion mutants (N-terminus, GST-Nef1-60; C-terminus, GST-Nef55-206) were produced and assayed for their ability to associate with eEF1A in vitro (Figure 2b). The GST-Nef160 and GST-Nef55-206 mutants had intact N-terminal and C-terminal sequences (data not shown). Lysates from MDMs were used in binding reactions with GST-HIV-1 Nef constructs. Of the three GST-Nef constructs, only GSTNefWT and GST-Nef55-206 were capable of binding eEF1A (Figure $2 \mathrm{~b}$ ). These results demonstrate that the $\mathrm{N}$-terminus of Nef is not required for eEF1A binding, and that the Nef fragment encoding amino acids $55-206$ is sufficient.

\section{Cytoplasmic relocalization of eEF1A by HIV-1 Nef is} mediated by Exp-t. The eEF1A protein is found in both the nucleus and cytoplasm of cells, which favors the nuclear export of mature tRNAs. In the absence of Nef, we observed relatively more eEF1A in the nucleus than the cytoplasm of primary MDMs (Figure 3a, mock). To determine whether Nef, via its interaction with eEF1A, interferes with the nuclear-cytoplasmic distribution of eEF1A, we treated
MDMs with rNef. ${ }^{25}$ Nuclear and cytoplasmic extracts were prepared from cells treated up to $12 \mathrm{~h}$ with $\mathrm{rNef}$, and eEF1A, Exp-t and rNef were assessed in both cellular compartments. rNef enhanced the nuclear-cytoplasmic transport of eEF1A up to $12 \mathrm{~h}$ post treatment in MDMs (Figure 3a). Our results indicate that $r$ Nef could actively favor the nuclear export of eEF1A in MDMs.

We tested several exportins, Exp-t, Exp-1, and Exp-5, in regard to the nuclear-cytoplasmic transport of eEF1A in the presence of rNef. Using MDM lysates, we detected a direct interaction between GST-Nef and Exp-t using a pull-down assay (Figure 3b). No direct interaction between GST-Nef and Exp-1 was observed (data not shown). Using lysates from untreated MDMs, we detected an interaction between eEF1A and Exp-t by co-immunoprecipitation (Figure 3c). We did not detect any interaction between eEF1A and Exp-1 in untreated MDMs (data not shown). Confirming a potential interaction between Exp-t, eEF1A and Nef, we observed an increase in eEF1A and Exp-t in the cytoplasmic extracts from MDMs treated with rNef as early as $30 \mathrm{~min}$ post treatment (Figure 3a). A sustained presence of $r$ Nef and eEF1A was detected in the cytoplasm of rNef-treated MDMs up to $12 \mathrm{~h}$ post treatment (Figure 3a). To determine the role of Exp-t in the nuclear-cytoplasmic transport of rNef/eEF1A complexes, MDMs were transfected with Exp-t siRNA $48 \mathrm{~h}$ before treatment with rNef. Knockdown of the Exp-t protein in MDMs was monitored by western blot (Figure 3d). Knockdown of Exp-t resulted in enhanced nuclear retention of eEF1A and rNef in MDM cultures treated with rNef (Figure 3e). We did not detect any nuclear retention of $\mathrm{rNef}$ or eEF1A following treatment of MDMs with the Exp-1 inhibitor leptomycin B or Exp-5 siRNA (data not shown). Our results indicate that, in rNef-treated MDMs, the nuclear-cytoplasmic transport of rNef/eEF1A complexes is preferentially mediated by Exp-t.

Nuclear-cytoplasmic relocalization of eEF1A by rNef blocks stress-induced apoptosis in macrophages. Brefeldin A (BFA) disrupts the organization of the microtubule and actin cytoskeleton and exerts ER and Golgi stress, resulting in apoptosis (Supplementary Figure 1). Interestingly, eEF1A is an actin/microtubule-binding protein and could interfere with BFA-induced apoptosis. Therefore, we examined whether nuclear-cytoplasmic transport of eEF1A by rNef inhibits BFA-induced apoptosis in MDMs, potentially due to cytoplasmic accumulation of eEF1A. We observed that pretreatment with rNef inhibits BFA-induced apoptosis in MDMs in a dose-dependent manner (Figure 4a). In addition, pretreatment with rNef did not inhibit apoptosis induced by tunicamycin (TM) or thapsigargin (TG) that disturb protein glycosylation or $\mathrm{Ca} 2+$ signaling, respectively (Figure 4b). Of the three Nef proteins, only NefWT and Nef55-206 were capable of blocking BFA-induced apoptosis in MDMs (Figure 4c).

Because Exp-t is involved in nuclear-cytoplasmic transportation of the eEF1A/rNef complexes, we assessed apoptosis among MDMs treated with rNef before BFA treatment and transfected with siRNA Exp-t, siRNA Exp-1, siRNA Exp-5, or a scrambled control. We observed that transfection with siRNA Exp-t, but not siRNA Exp-1 or siRNA Exp-5, inhibited the rNef-mediated blockade of apoptosis in 


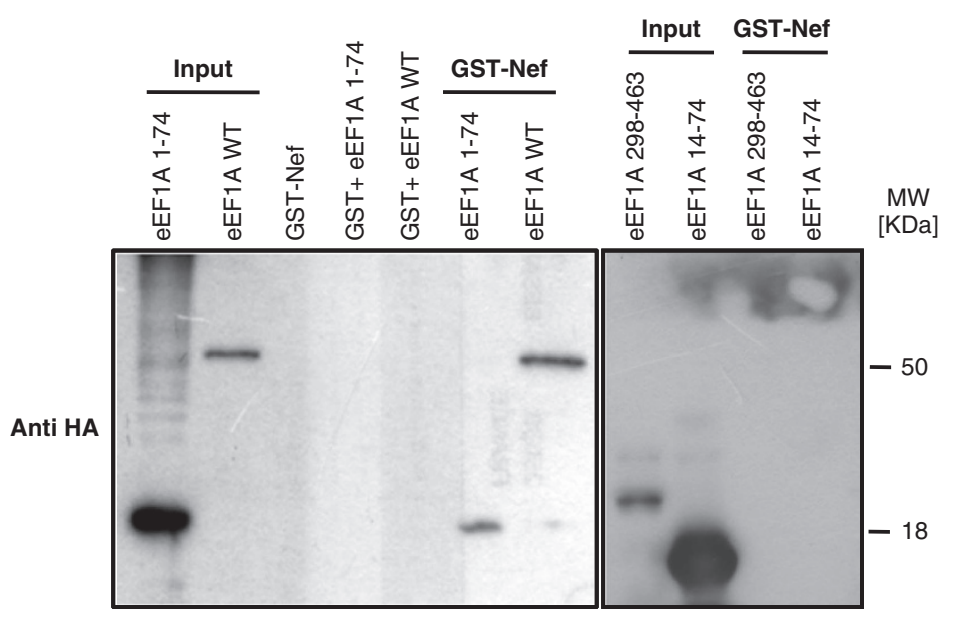

b
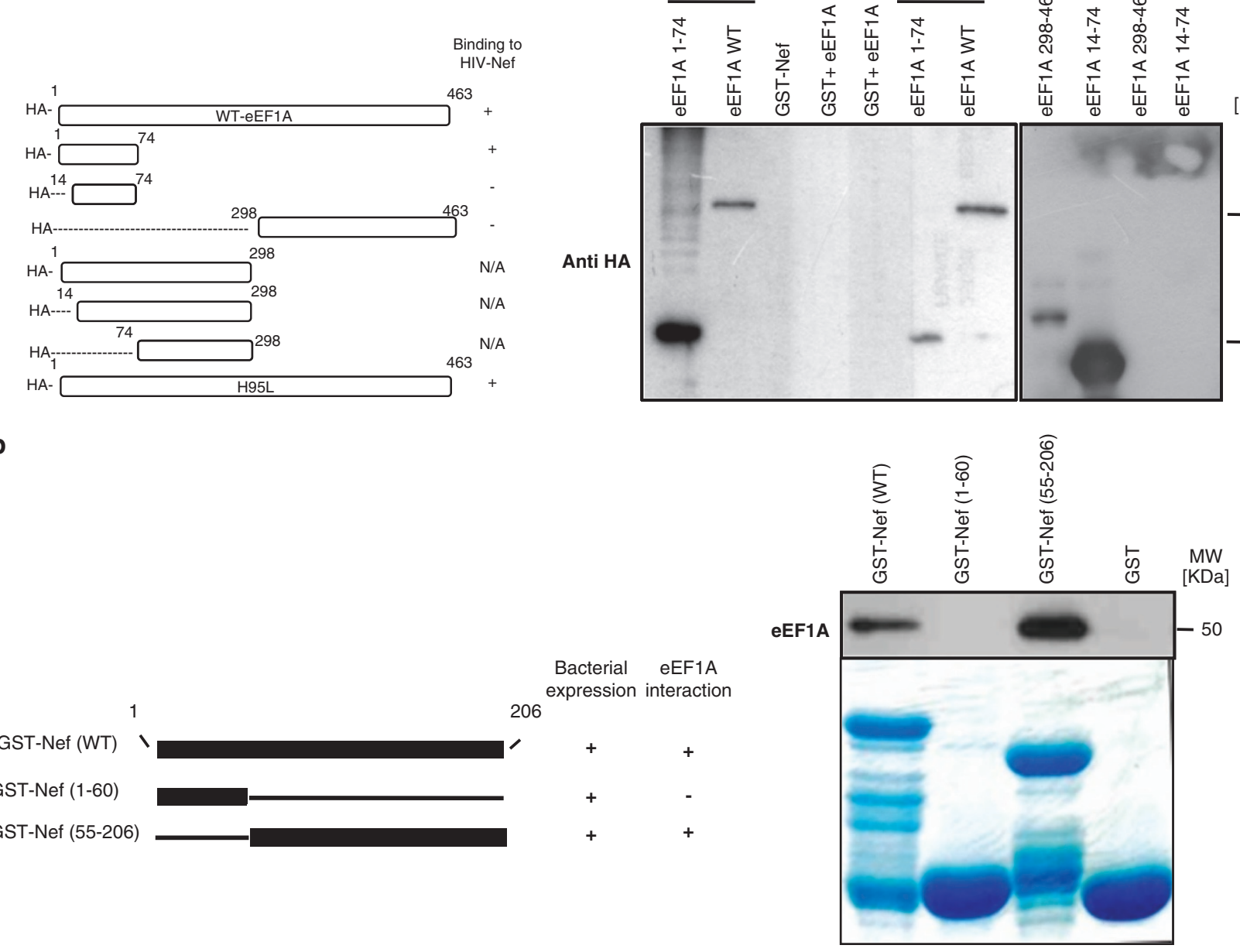

Figure 2 The N-terminal 74 amino acids of eEF1A bind to the C-terminal region (aa 55-206) of HIV-1 Nef. (a) The N-terminal region of eEF1A is sufficient for binding to HIV-1 Nef. Left panel: schematic diagram of eEF1A mutants expressed in bacteria as HA-tagged fusion proteins ${ }^{24}$ and their Nef-binding properties. Numbers indicate the amino-acid residues retained by the deletion mutants. In vitro Nef binding of these mutants are reported qualitatively as + or - to the right of the figure. N/A, not applicable; WT, wild type. Right panel: eEF1A interacts with HIV-1 Nef via its N-terminus extremity (aa 1-74). Using wild-type GST-Nef constructs, binding of purified WT eEF1A, eEF1A 1-74, eEF1A 14-74 and eEF1A 298-463 were measured in GST pull-down assays. Input corresponds to $10 \%$ of the material used for pull-down. Results are representative of two independent experiments. (b) The C-terminal region (aa 55-206) of HIV-1 Nef is sufficient for binding to eEF1A. Left panel: schematic diagram of WT HIV-1 Nef and mutants expressed in bacteria as GST-tagged fusion proteins. The names of the mutants are shown to the left of the figure; numbers refer to the amino-acid residues retained by the deletion mutants. The bacterial expression status and in vitro binding of these mutants are reported qualitatively as + or - to the right of the figure. Right panel represents the interaction of HIV-1 Nef with eEF1A via its C-terminal region (aa 55-206) and coomassie staining of expressed proteins that were used in the binding reaction of GST pull-down. The binding of eEF1A present in lysates of MDMs was measured in GST pull-down assays using WT and mutated GST-Nef constructs. Results are representative of two independent experiments

MDMs treated with BFA (Figure 4d). Furthermore, the knockdown of eEF1A by siRNA inhibited the Nef-mediated blockade of apoptosis in MDMs treated with BFA (Figure 4e). Our results indicate that Exp-t-mediated nuclear-cytoplasmic transport of the rNef/eEF1A complexes in MDMs is involved in the inhibition of apoptosis triggered by stress, such as BFA treatment.

As shown in Figure 5a, p35 pro-caspase- 3 is diminished and cleaved to $\mathrm{p} 20 / \mathrm{p} 17$ proteins in MDMs treated with BFA, confirming that BFA-induced apoptosis activates caspase$3^{26,27}$ Under ER stress treatments, such as BFA, the magnitude of protection against apoptosis directly correlates with the level of cytoplasmic eEF1A expression; ${ }^{28,29}$ therefore, we measured the levels of eEF1A in the cytosol of BFA- stimulated MDMs treated with increasing concentrations of Nef. We observed that the levels of cytosolic eEF1A positively correlated with Nef treatment in a dose-dependent manner, as well as with the resistance to apoptosis in BFA-stimulated MDMs as measured by decreased caspase- 3 activation and cytochrome $c$ release (Figure $5 b$ ).

Cytoplasmic extracts of BFA-stimulated MDMs treated with rNef, in the absence or presence of Exp-t siRNA, Exp-1 siRNA, or Exp-5 siRNA, were analyzed for caspase-3 activation. Exp-t, Exp-1, and Exp-5 knockdown in MDMs was monitored by western blot (Figures 3d and 5c). Knockdown of Exp-t, but not Exp-1 or Exp-5, relieved the Nefmediated blockade of apoptosis in BFA-stimulated MDMs (Figure $5 \mathrm{~d}$ ). Relief of the Nef-mediated blockade of apoptosis 
a

Nef
Cytoplasm

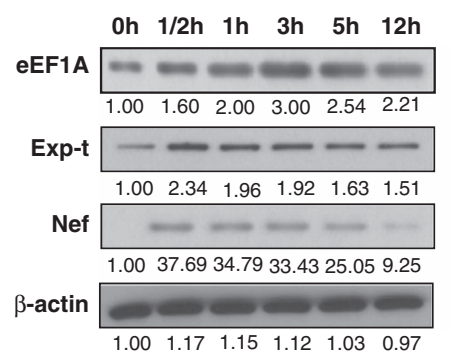

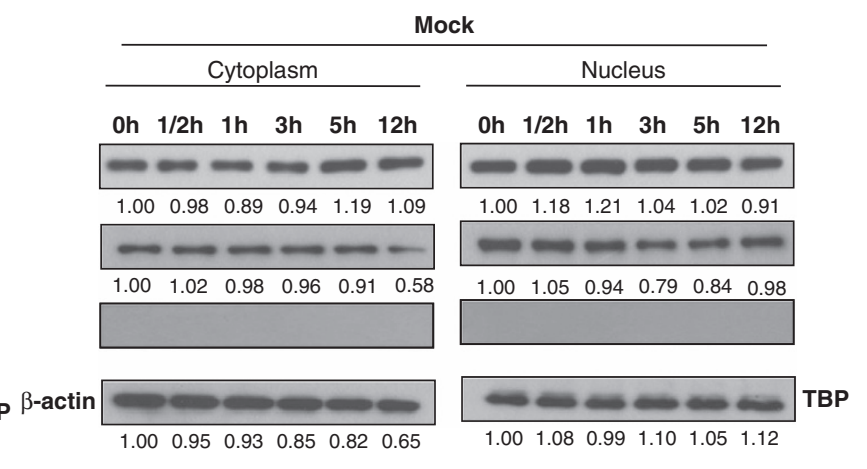
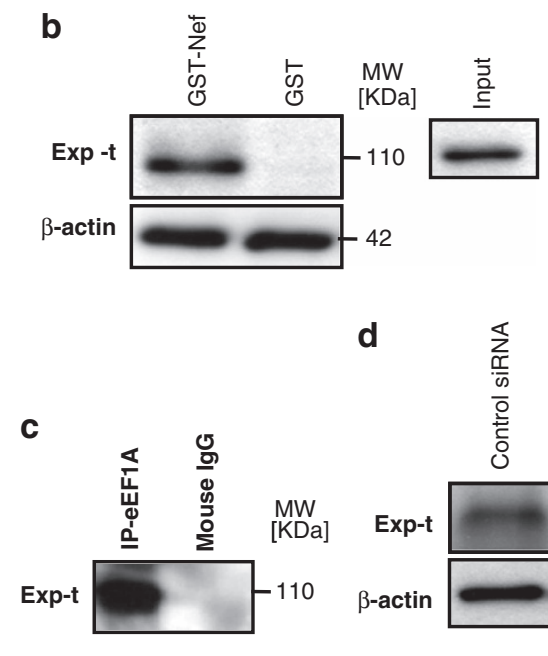

d

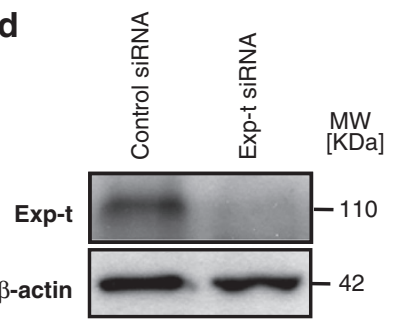

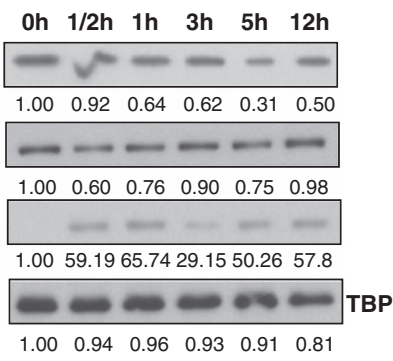

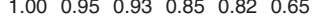

$\begin{array}{llllll}1.00 & 1.08 & 0.99 & 1.10 & 1.05 & 1.12\end{array}$

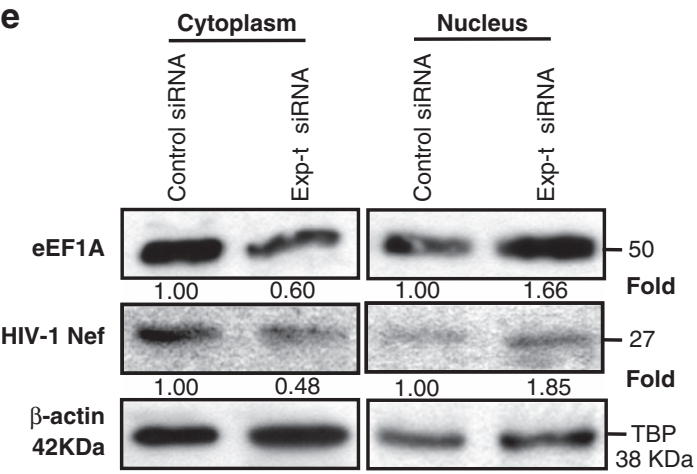

Figure 3 Nuclear-cytoplasmic relocalization of eEF1A/rNef occurs in MDMs treated with rNef and depends on Exp-t. (a) Kinetics of eEF1A/rNef/Exp-t in nuclear and cytoplasmic compartments of MDMs. Nuclear and cytoplasmic extracts of MDMs treated with rNef (100 ng/ml) were prepared and the expression of eEF1A, Exp-t and rNef were assessed in both cellular compartments up to $12 \mathrm{~h}$ post treatment using western blotting. Similarly, nuclear and cytoplasmic extracts of untreated MDMs (mock) were prepared and tested for the expression of eEF1A, Exp-t and rNef. $\beta$-actin and TBP were used as loading controls. Results are representative of three independent experiments. (b) Using wild-type GST-Nef constructs, the binding of Exp-t present in MDM lysates was assessed in GST pull-down assays. Input corresponds to $10 \%$ of the material used for pull-down. Results are representative of two independent experiments. (c) eEF1A interacts with Exp-t in MDM lysates. Total MDM extracts were prepared and the eEF1A/Exp-t interaction assessed by immunoprecipitation with an anti-eEF1A antibody and western blotting with an anti-Exp-t monoclonal antibody. Results are representative of three independent experiments. (d) Knockdown of the Exp-t protein by siRNA in MDMs. MDM cultures were transfected with a scrambled control or Exp-t siRNA and total cellular extracts were prepared $48 \mathrm{~h}$ post transfection. Protein expression was analyzed by western blot. $\beta$-actin was used as a loading control. (e) Effect of Exp-t siRNA on nuclear-cytoplasmic transport of eEF1A and rNef in MDMs. MDM cultures were transfected with a scrambled control or Exp-t siRNA for $48 \mathrm{~h}$ before treatment with $\mathrm{rNef}(100 \mathrm{ng} / \mathrm{ml})$ for $3 \mathrm{~h}$. Nuclear and cytoplasmic extracts were prepared and analyzed by western blot using anti-Nef and anti-eEF1A antibodies. $\beta$-actin and TBP were used as loading controls. Results are representative of two independent experiments. Protein levels of eEF1A and Nef after siRNA transfection were quantified by densitometry using ImageJ 1.40 software (protein levels in cells transfected with scrambled siRNAs were arbitrarily established at 1)

Figure 4 rNef-mediated inhibition of BFA-induced apoptosis in MDMs parallels cytoplasmic accumulation of eEF1A and is dependent on Exp-t. (a) rNef prevents BFAinduced apoptosis in MDMs. MDMs were treated with BFA $(10 \mu \mathrm{g} / \mathrm{ml})$ for 12 or $15 \mathrm{~h}$ or left untreated in the presence of increasing concentrations of $\mathrm{rNef}(0,125,750 \mathrm{ng} / \mathrm{ml})$. Apoptosis was detected by annexin-V flow cytometric analysis. The histogram summarizes the survival of MDMs following treatment with BFA (10 $\mu \mathrm{g} / \mathrm{ml})$ for 12 or $15 \mathrm{~h}$ in the presence of increasing concentrations of rNef. The results represent means of three independent experiments. ${ }^{*} P<0.05$. (b) rNef prevents BFA-induced apoptosis, but neither TM-induced apoptosis nor TG-induced apoptosis in MDMs. MDMs were treated with BFA (10 $\mu \mathrm{g} / \mathrm{ml}), \mathrm{TM}(10 \mu \mathrm{g} / \mathrm{ml})$ or TG $(10 \mu \mathrm{g} / \mathrm{ml})$ for 5 or $12 \mathrm{~h}$ or left untreated in the presence of $\mathrm{rNef}(1 \mu \mathrm{g} / \mathrm{ml})$. Apoptosis was measured by the TUNEL assay. Results are representative of data observed in three independent experiments. The mock panel is shown in triplicate to the left of the BFA, TM and TG panels to facilitate the results reading. (c) The C-terminal extremity of Nef prevents BFA-induced apoptosis in MDMs. MDMs were treated with BFA (10 $\mu \mathrm{g} / \mathrm{ml})$ for $12 \mathrm{~h}$ or left untreated in the presence of WTNef, Nef1-60 or Nef55-206 $(1 \mu \mathrm{g} / \mathrm{ml})$. Apoptosis was measured by the TUNEL assay. Results are representative of data observed in two independent experiments. (d) Blockade of BFA-induced apoptosis in MDMs by rNef is dependent on Exp-t. MDM cultures were transfected with a scrambled control, Exp-t siRNA, Exp-1 siRNA, or Exp-5 siRNA for $48 \mathrm{~h}$ before treatment, with BFA $(10 \mu \mathrm{g} / \mathrm{ml})$ for $12 \mathrm{~h}$ or left untreated in the presence $(1 \mu \mathrm{g} / \mathrm{ml})$ or absence of $r$ Nef. Apoptosis was detected by flow cytometric analysis of annexin-V. The histogram shows the survival percentage of MDMs treated with mock, BFA, or BFA + Nef in the presence of exportin siRNAs. Results are representative of three independent experiments. ${ }^{*} P<0.05$. (e) Blockage of BFA-induced apoptosis in MDMs by rNef is dependent on eEF1A. MDMs were treated with control siRNA or eEF1A siRNA for $48 \mathrm{~h}$ before treatment, with BFA ( $10 \mu \mathrm{g} / \mathrm{ml})$ for $12 \mathrm{~h}$ or left untreated in the presence $(1 \mu \mathrm{g} / \mathrm{ml})$ or absence of rNef. Apoptosis was measured by the TUNEL assay. Results are representative of two independent experiments. EF1A knockdown in MDMs was monitored by western blot (upper right panel). The histogram shows the percentage of TdT positive cells 
a
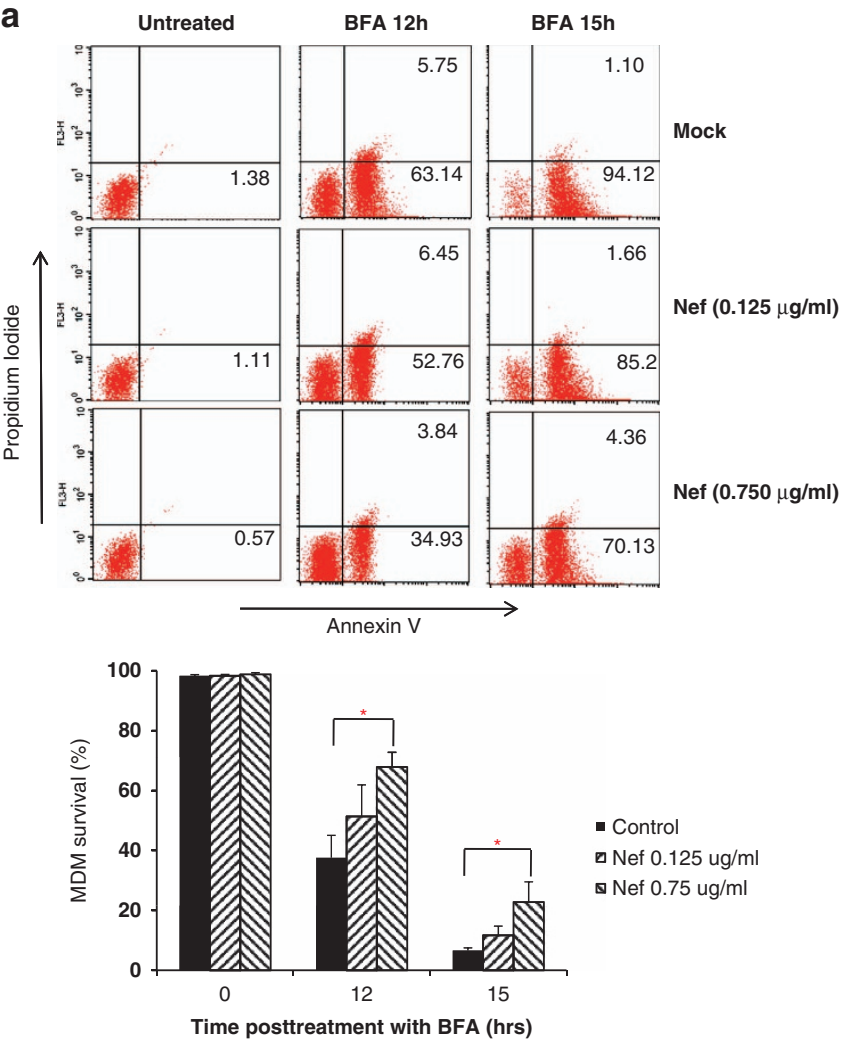

b

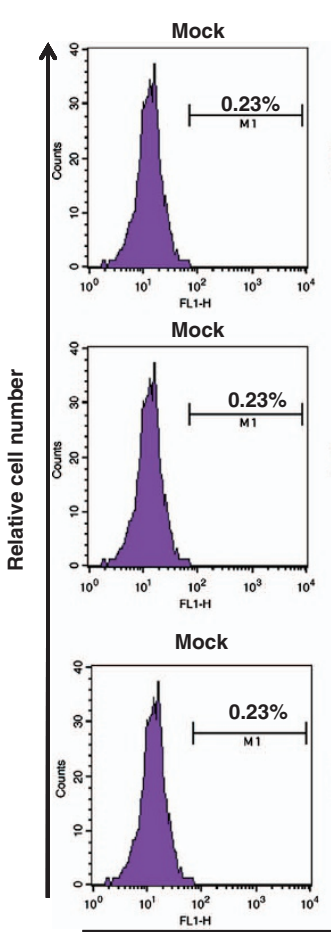

$5 \mathrm{H}$

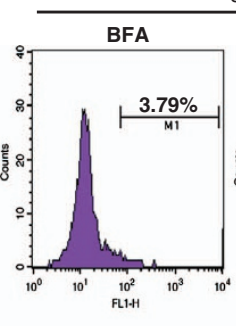

TM

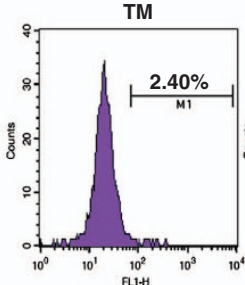

TG

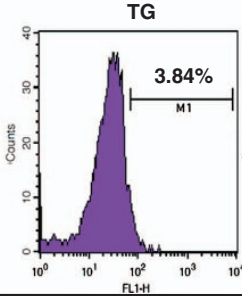

BFA+Nef

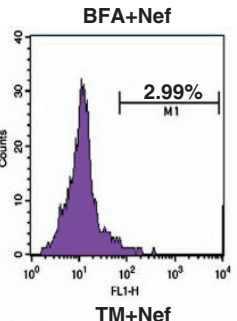

TM+Nef

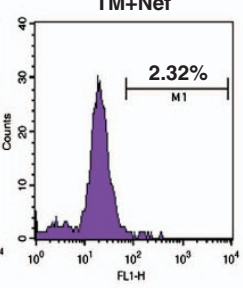

TG+Nef

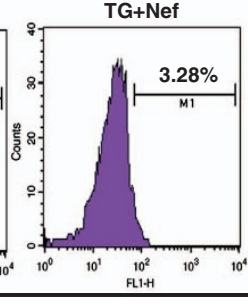

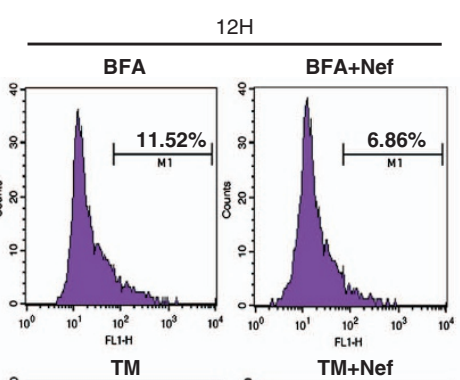
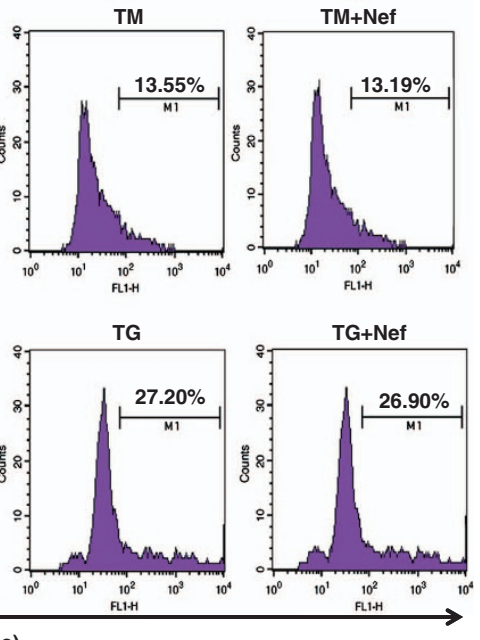

TdT Labeling (log fluorescence)

Figure 4 (Continued) 
c
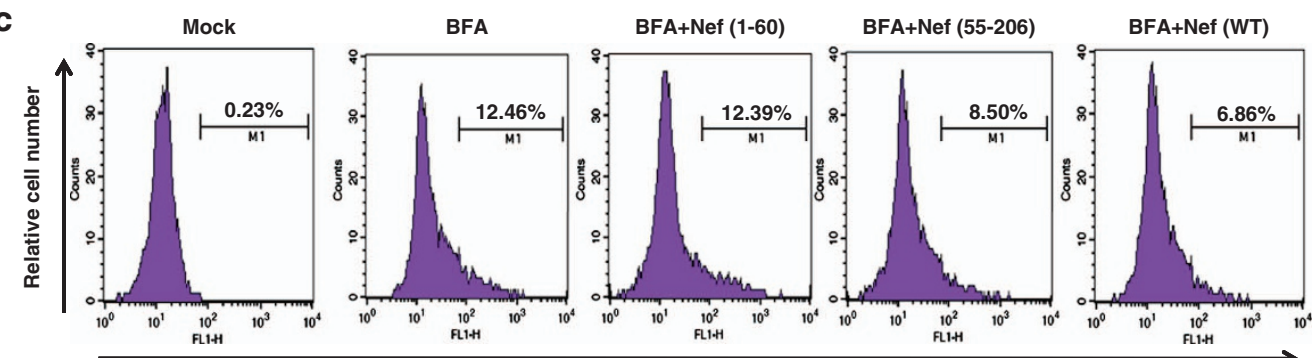

TdT Labeling (log fluorescence)

d
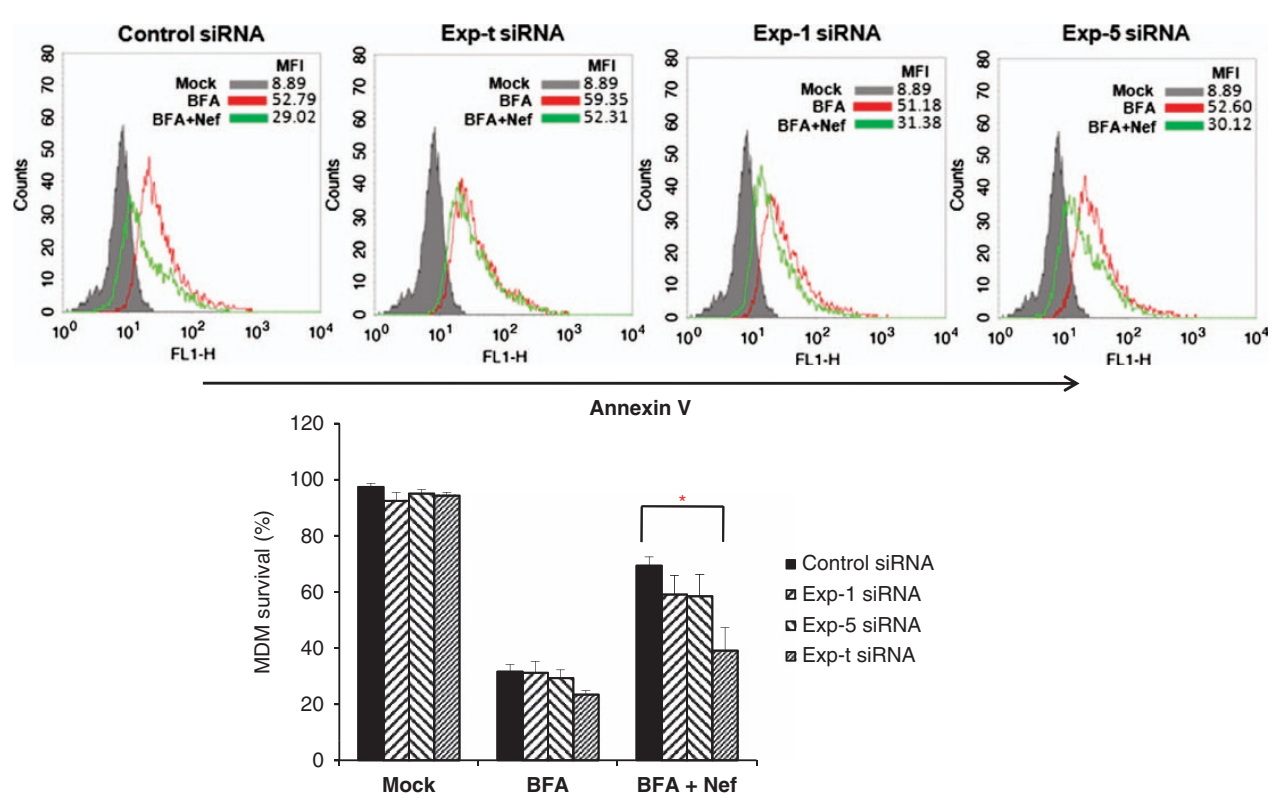

e
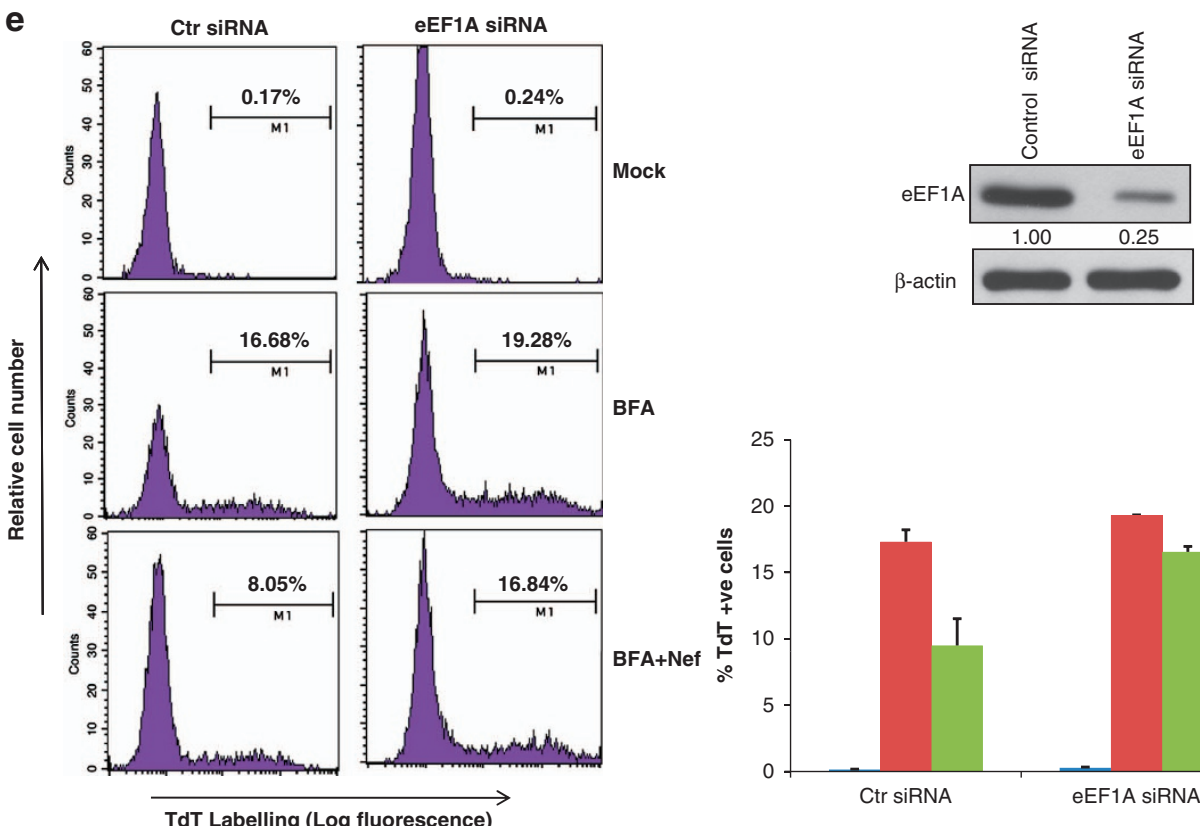

Figure 4 (Continued) 
in BFA-stimulated MDMs transfected with Exp-t siRNA was accompanied by decreased cytoplasmic eEF1A (Figure 5d). Our results indicate a critical role for Exp-t and eEF1A in resistance to apoptosis in BFA-treated MDMs.

Cytochrome $c$-induced caspase- 9 activation is regulated at multiple levels, and tRNA was reported recently to bind to cytochrome $c$, blocking formation of the apoptosome and the subsequent activation of caspase-9. ${ }^{30}$ Therefore, we tested whether Nef can prevent cytochrome $c$ release in the cytoplasm. We found that most of the cytochrome $c$ was detected in the mitochondrial fraction from normal MDMs, whereas the majority of the cytochrome $c$ in BFA-treated MDMs was detected in the cytosolic fraction (Figure 5e). In Nef-treated MDMs, most of the cytochrome $c$ was detected in the mitochondrial fractions, even after BFA stimulation, which suggests that mitochondrial cytochrome $c$ release is blocked by Nef treatment (Figure 5e).

As shown in Figure 5f, cleavage of 45-kDa procaspase- 9 to a 35-kDa fragment in MDMs treated with BFA was blocked by Nef, but not by inactivated boiled Nef, confirming that BFAinduced apoptosis activates caspase- 9 and that it can be inhibited by Nef. The processing of caspase- 9 induced by BFA in Nef-treated MDMs was inhibited in a dose-dependent manner (Figure $5 \mathrm{~g}$ ), indicating that Nef may function by preventing the activation of caspase- 9 via decreased release of mitochondrial cytochrome c. As shown in Figure 5h, pretreatment with $\mathrm{rNef}$ did not inhibit the processing of caspase- 3 and caspase- 9 induced by TM or TG.

eEF1A/Nef complexes contain tRNAs and block stressinduced apoptosis in MDMs through tRNA binding to cytochrome $\boldsymbol{c}$. As both eEF1A and HIV-1 Nef bind to tRNAs preferentially, ${ }^{31}$ we specifically assessed the presence of several tRNAs in the rNef/eEF1A complexes in MDMs treated with rNef. MDMs treated with $r$ Nef were lysed and the presence of tRNAs in the immunoprecipitated eEF1A/ rNef complexes was analyzed using RT-PCR. We detected the presence of all tested tRNAs, namely tRNAMet, tRNAPhe, tRNATrp, and tRNALys in the eEF1A/rNef complexes in MDMs (Figures $6 \mathrm{a}, \mathrm{c}$ and $\mathrm{d}$ ). Furthermore, the knockdown of eEF1A by siRNA resulted in the partial inhibition of tRNA binding to cytochrome $c$ (Figure $6 \mathrm{~b}$ ). The $\Delta \Delta \mathrm{Ct}$ values were determined separately for untreated cells and averaged around 1 (Supplementary Figure 2).

The binding of tRNAs to cytochrome $c$ was recently reported to inhibit caspase- 9 activation. Therefore, we hypothesized that the enhanced nuclear-cytoplasmic transport of eEF1A/rNef/tRNA complexes mediated by Exp-t might result in higher levels of cytosolic tRNAs, which could target cytochrome $c$ released in BFA-treated MDMs. Cytoplasmic extracts of MDMs treated with increasing amounts of rNef were tested for the presence of tRNAs complexed with immunoprecipitated eEF1A and cytochrome $c$. We observed that tRNA binds to eEF1A in the cytosol of BFA-treated MDMs in a Nef-dependent manner (Figure 6e). Similar results were obtained for the binding of tRNA to cytochrome $c$ (Figure 6f), indicating that Nef treatment of BFA-stimulated MDMs favors the formation of tRNA/cytochrome $c$ complexes in the cytoplasm. Cytoplasmic extracts of BFA-stimulated MDMs treated with rNef, in the absence or presence of Exp-t siRNA,
Exp-1 siRNA, or Exp-5 siRNA, were analyzed for the presence of tRNA binding cytochrome $c$. Knockdown of Exp-t, but not Exp-1 or Exp-5, decreased the amount of tRNAs that bound to cytochrome $c$, as well as eEF1A, in BFAstimulated MDMs treated with Nef (Figures $6 g$ and $h$ ). Similarly, the knockdown of eEF1A by siRNA decreased the amount of tRNAs that were associated with cytochrome $c$ in BFA-stimulated MDMs treated with Nef (Figure 6i).

\section{Discussion}

We observed a direct interaction between HIV-1 Nef and eEF1A. In rNef-treated MDMs, the rNef-mediated nuclearcytoplasmic relocalization of eEF1A was dependent on Exp-t. We also report the specific presence of tRNAs in the rNef/eEF1A complexes. Nuclear-cytoplasmic relocalization of $r$ Nef/eEF1A/tRNA prevented stress-induced apoptosis of MDMs treated with BFA through decreased release of mitochondrial cytochrome $c$, enhanced tRNA binding to release cytosolic cytochrome $c$, and increased cytoplasmic levels of eEF1A, ultimately resulting in decreased caspase activation (Figure 7). Our results indicate that the HIV-1 Nef protein enhances resistance to stress-induced apoptosis in MDMs through the nuclear-cytoplasmic relocalization of rNef/ eEF1A/tRNA complexes.

Our results show that nuclear-cytoplasmic transport of eEF1A/Exp-t/tRNA complexes occurs in MDMs treated with rNef. Although the role of eEF1A in tRNA transport has been reported, this is the first time that a viral protein such as Nef has been shown to be actively involved in such a process. The presence of relatively more amounts of eEF1A in the nucleus of MDMs confirms previous results indicating that eEF1A is localized in both the cytoplasm and nucleus of cells. We also observed that the nuclearcytoplasmic distribution of eEF1A/rNef complexes included the presence of RNA (Figures $6 a-d)$. Both proteins most probably bind to each other in an RNA-independent manner. First, we observed the interaction between Nef and eEF1A in a purified system (Figure 2a). Second, supportive experimental evidence comes from crosslinking studies between rabbit EF-1 $\alpha$ GTP and aminoacyl-tRNA that indicate contact sites corresponding to residues 270-275, 345-355 and 375 , sites that are in contact with the RNA in the ternary complex crystal. ${ }^{32}$ Finally, the N-terminal Arg-rich region of HIV-1 Nef (aa 1-22) binds to RNA in vitro. ${ }^{31}$ As the Nef fragment encoding amino acids 55-206 is sufficient for binding to the N-terminal extremity of eEF1A (Figure $2 b$ ), it is highly unlikely that the Nef interaction with eEF1A requires the presence of RNA.

We hypothesized that tRNA is the most likely candidate to be present in the Nef-eEF1A complex because eEF1A binds to aminoacyl-tRNA during translation and Nef has been shown to bind to tRNAs and promote the annealing of primer tRNA to viral genomic RNA. In addition, Exp-t, rather than Exp-5, has a critical role in this process, which is in agreement with a critical role of Exp-t in the selection and transport of mature tRNAs from the nucleus to the cytoplasm.

Nef has been reported to prevent both Fas and TNF receptor-mediated deaths in HIV-infected T cells via interaction with ASK-1, thereby blocking the extrinsic apoptosis 
a

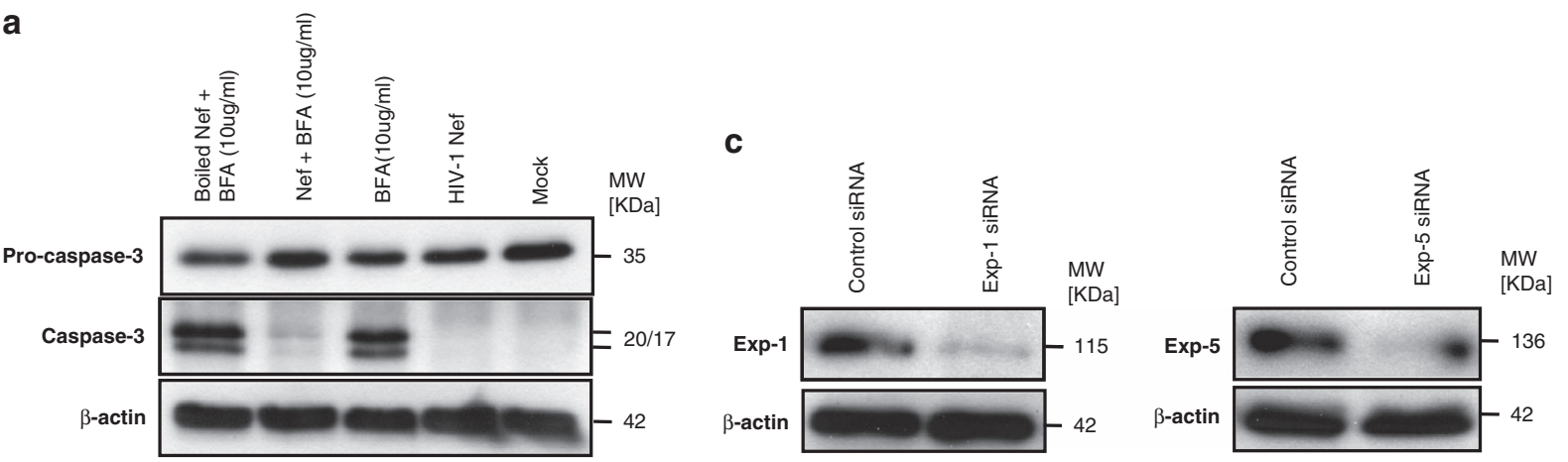

b
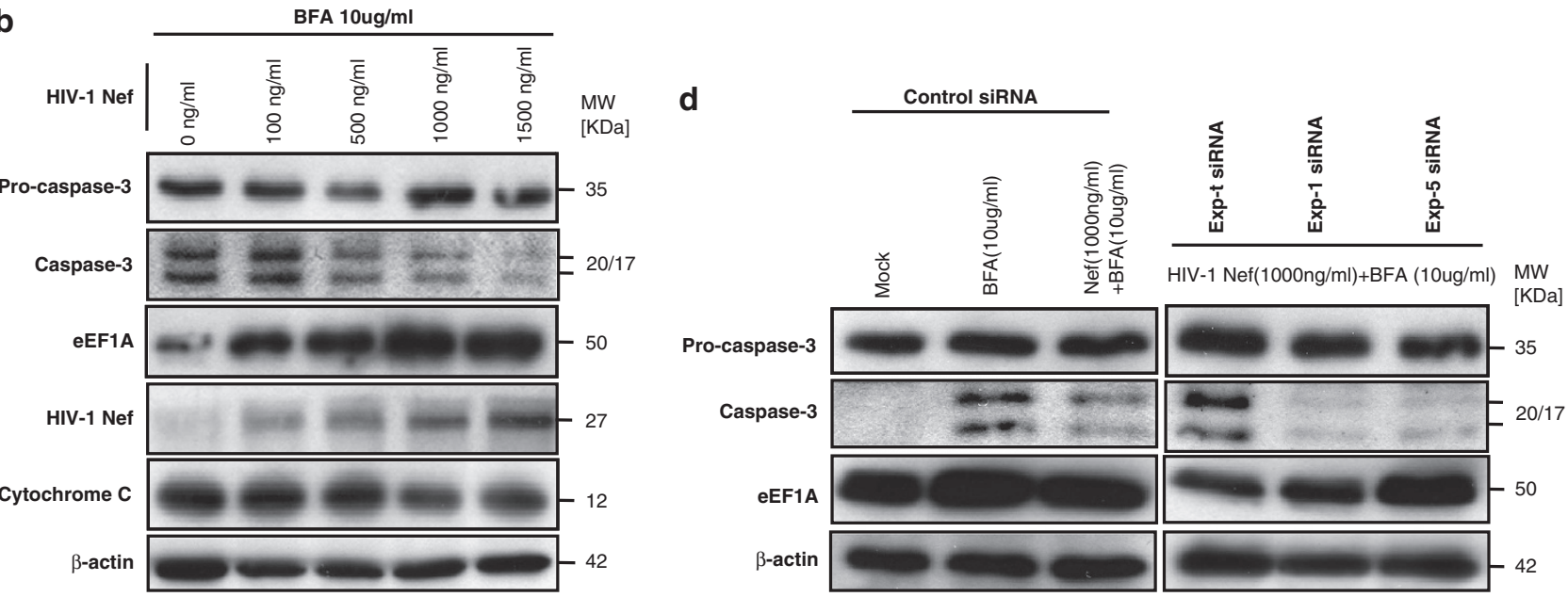

e

Cyto fraction

Mito fraction
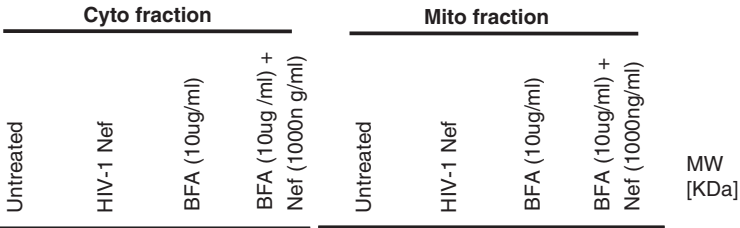

Cytochromec
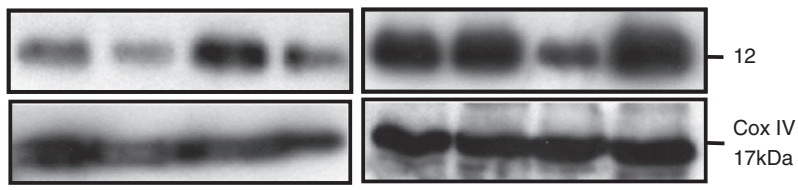

f

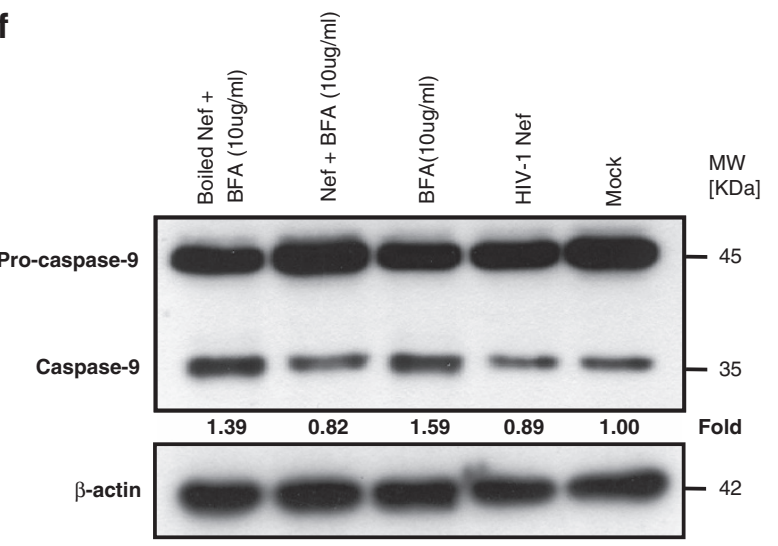

Figure 5 (Continued) g

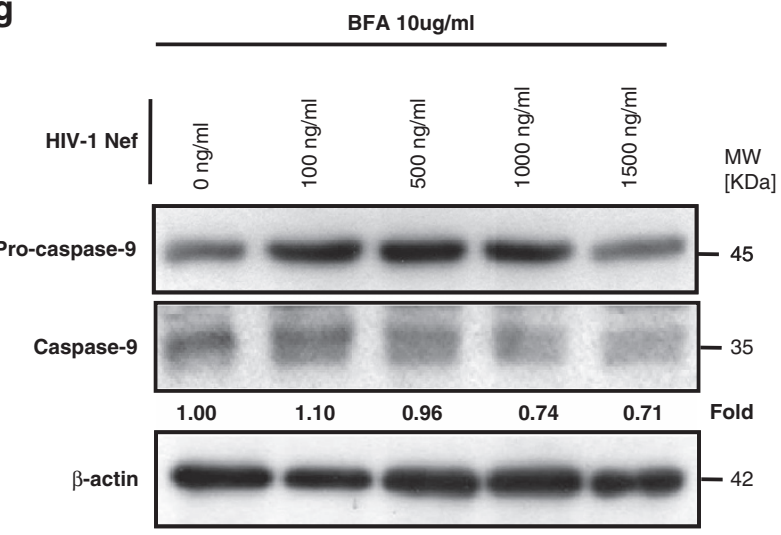


h
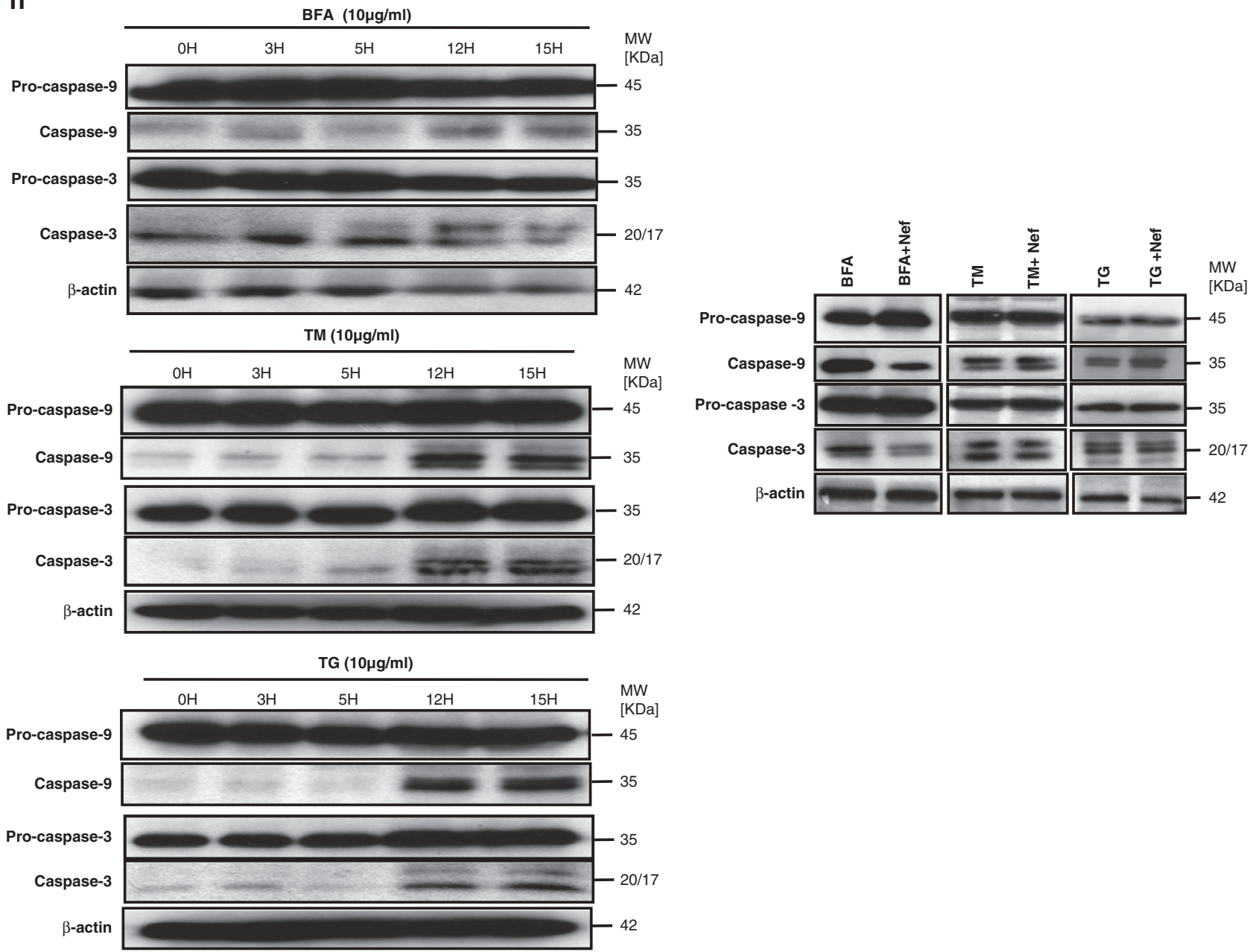

Figure 5 rNef-mediated cytoplasmic accumulation of eEF1A in BFA-treated MDMs inhibits caspase activation and decreases the cytoplasmic release of cytochrome $c$. (a) Inhibition of caspase-3 activation in BFA-stimulated MDMs treated with $\mathrm{rNef}(1000 \mathrm{ng} / \mathrm{ml})$. (b) Inhibition of caspase-3 activation in BFA-stimulated MDMs treated with rNef is dose-dependent. Mitochondrial cytochrome $c$ release in BFA-treated MDMs is blocked by rNef in a dose-dependent manner and positively correlates with cytoplasmic accumulation of eEF1A. (c) Knockdown of Exp-1 and Exp-5 proteins by siRNA in MDMs. MDM cultures were transfected with a scrambled control, Exp-1 siRNA, or Exp-5 siRNA. Total cellular extracts were prepared $48 \mathrm{~h}$ post transfection. Protein expression was analyzed by western blot. $\beta$-actin was used as a loading control. (d) Inhibition of caspase-3 activation in BFA-stimulated MDMs treated with rNef is dependent on Exp-t. (e) Mitochondrial cytochrome $c$ release in BFA-treated MDMs is blocked by rNef. (f) Inhibition of caspase-9 activation in BFA-stimulated MDMs treated with $\mathrm{rNef}(1000 \mathrm{ng} / \mathrm{ml})$. Protein levels of caspase-9 were quantified by densitometry using ImageJ 1.40 software (the level of caspase-9 in mock cells was arbitrarily established at 1). (g) Inhibition of caspase-9 activation in BFA-stimulated MDMs treated with rNef is dosedependent. Protein levels of caspase-9 were quantified by densitometry using ImageJ 1.40 software (the level of caspase-9 in mock cells was arbitrarily established at 1). (h) Inhibition of caspase activation in BFA-stimulated MDMs treated with rNef, but neither in TM-stimulated MDMs nor in TG-stimulated MDMs treated with rNef. Left panel: time-course of caspase activation in BFA-stimulated MDMs, TM-stimulated MDMs or TG-stimulated MDMs. Right panel: inhibition of caspase activation in BFA-stimulated MDMs treated with rNef, but neither in TM-stimulated MDMs nor in TG-stimulated MDMs treated with rNef. MDM cultures were treated with BFA (10 $\mu \mathrm{g} / \mathrm{ml}), \mathrm{TM}(10 \mu \mathrm{g} / \mathrm{ml})$, or $\mathrm{TG}(10 \mu \mathrm{g} / \mathrm{ml})$ for $12 \mathrm{~h}$ in the presence of $\mathrm{rNef}(1 \mu \mathrm{g} / \mathrm{ml})$, and caspase-3 and -9 activation was measured in total cellular lysates. Results are representative of data obtained in three independent experiments

pathway. Nef inhibits ASK-1, caspase-3, and caspase-8 activation, resulting in the blockade of apoptosis in HIVinfected cells. Nef has also been shown to block the intrinsic apoptosis pathway via inhibition of the pro-apoptotic protein Bad. ${ }^{33}$ Our data highlight a new molecular mechanism elicited by the HIV-1 Nef protein to block the intrinsic apoptosis pathway in Nef-expressing cells, such as HIV-1-infected MDMs. The HIV-1 Nef protein, through the nuclearcytoplasmic relocalization of rNef/eEF1A/tRNA complexes, could participate in preventing stress-mediated effects at several levels. First, the decreased cytochrome $c$ release is probably due to cytoplasmic accumulation of eEF1A that stabilizes actin filaments and potentially could block the BFAinduced disruption of the organization of the microtubule and actin cytoskeleton. Second, the tRNAs could be specifically delivered by the eEF1A/Nef/tRNA complexes to cytosolic cytochrome $c$, thereby preventing cytochrome $c$ interaction with Apaf-1 and blocking caspase activation and apoptosis. Third, the eEF1A protein could be delivered by the eEF1A/ Nef/tRNA complexes in the cytoplasm to participate in cytoskeleton rearrangement aimed to eliminate unfolded proteins produced during stress events. ${ }^{34-36}$ Finally, eEF1A has been reported to activate Akt, the protein kinase $B$ reported by numerous studies to favor resistance to apoptosis. ${ }^{11}$ 
a

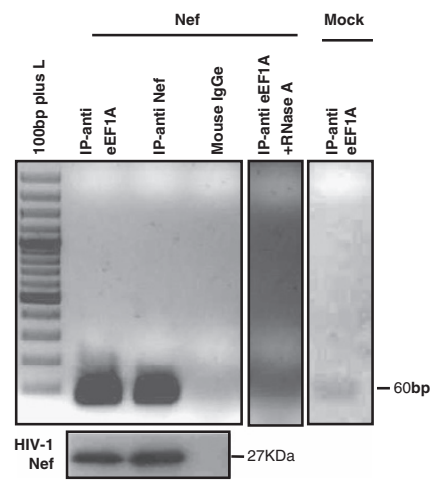

c

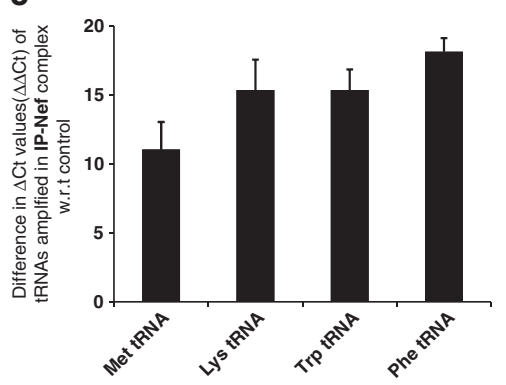

e

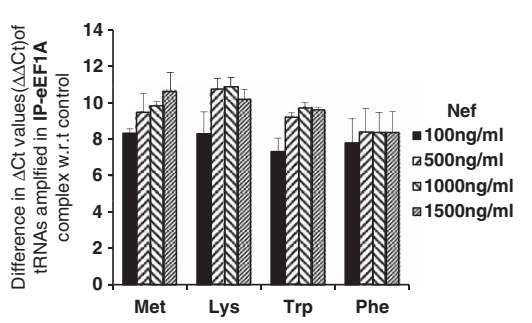

g

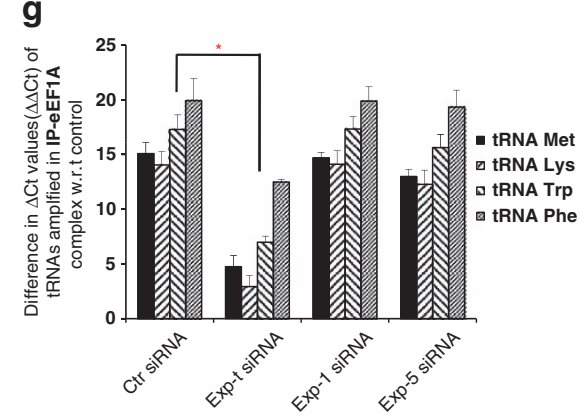

b

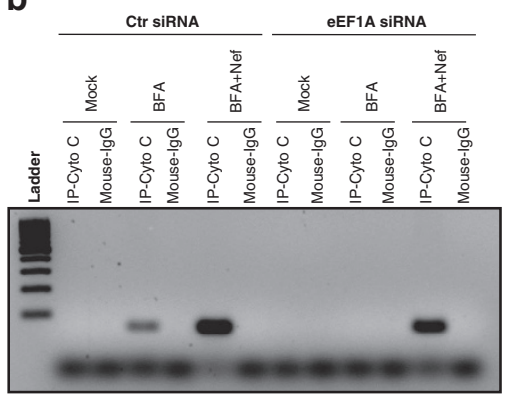

d

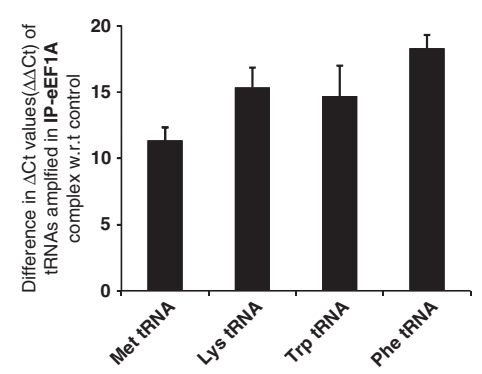

f

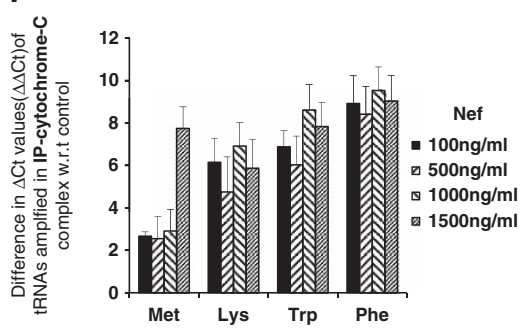

h

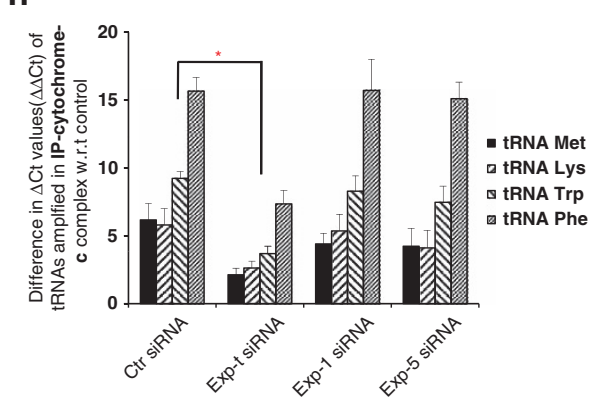

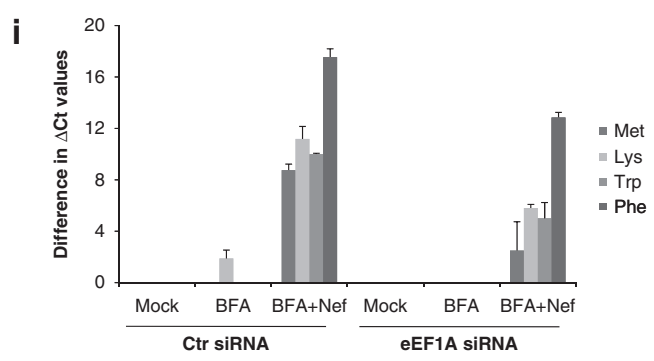


In order to determine the scope of eEF1A-mediated protection, we assayed eEF1A antiapoptotic activity in response to ER stress agents, BFA, TM and TG. BFA reversibly blocks protein transport between the ER and Golgi, whereas TM blocks the synthesis of all $\mathrm{N}$-linked glycoproteins (N-glycans) ${ }^{37}$ and TG inhibits the ER Ca2 + -ATPase family of calcium pumps. ${ }^{38}$ Our results indicate that Nef specifically blocks apoptosis induced by the ER-stress agent BFA, indicating that increased cytoplasmic relocalization of eEF1A by HIV-1 Nef may alleviate the inhibition of protein transport between the ER and Golgi observed in BFA-treated cells.
In addition, Nef could favor an homeostatic control of UPR and of oxidative stress observed during the acute phase of HIV infection and subsequently fuel the progression of the disease toward a chronic infection with the formation of cellular reservoirs of the virus.

Taken together, the results indicate that cytoplasmic relocalization of the eEF1A/tRNAs complexes by HIV-1 Nef could ultimately favor the survival of the cell under stress, thereby allowing optimal viral replication. This event may participate in the formation and maintenance of viral reservoirs in HIV-infected persons.

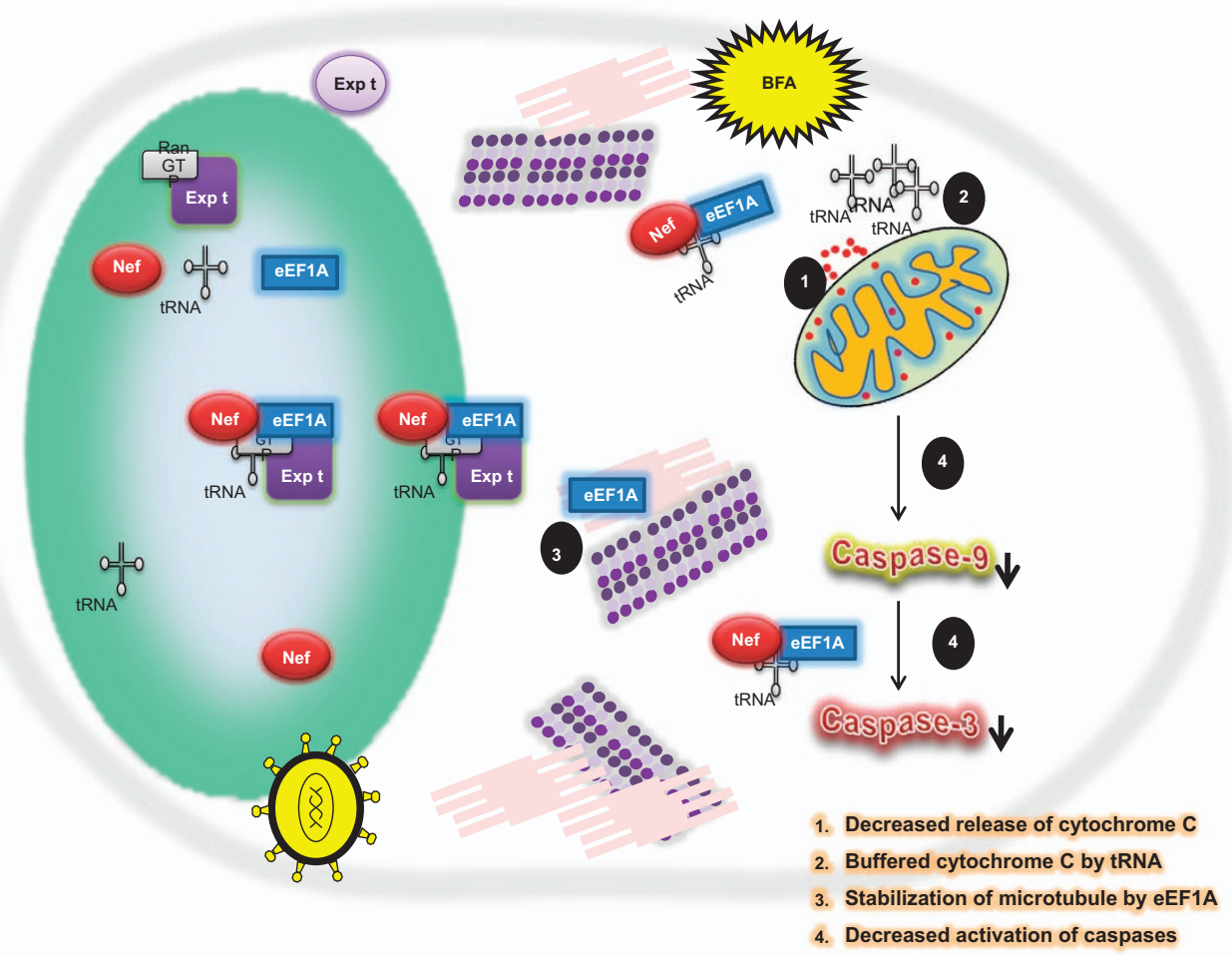

Figure 7 Potential inhibitory effect of the eEF1A/rNef/tRNA complex on the intrinsic apoptotic pathway in BFA-treated MDMs. HIV-1 Nef protein favors translocation and accumulation of eEF1A from the nucleus toward the cytoplasm of the cell. tRNA is present in the Nef/eEF1A complex that is exported by Exp-t and buffers the cytochrome $c$ released under oxidative stress conditions. At the same time the Nef/eEF1A complex inhibits caspase activation. Furthermore, eEF1A could stabilize the microtubules and give relief to the cell to survive under stress conditions. BFA, brefeldin A; Exp-t, exportin-t

Figure 6 rNef-mediated cytoplasmic accumulation of eEF1A in BFA-treated MDMs parallels tRNA binding to cytochrome c. (a) Direct detection of tRNAs in rNef/eEF1A complexes in MDMs treated with rNef. Lysates from MDMs treated with $\mathrm{rNef}(100 \mathrm{ng} / \mathrm{ml})$ for $3 \mathrm{~h}$ or left untreated (mock) were prepared, immunoprecipitated with anti-eEF1A and anti-Nef antibodies, and tRNALys in the eEF1A/rNef complexes was amplified by RT-PCR. Lysates were also immunoprecipitated with an isotype control antibody. The presence of HIV-1 Nef protein in the immune complex was determined by western blot. As an internal control, lysates from MDMs treated with $\mathrm{rNef}(100 \mathrm{ng} / \mathrm{ml})$ for $3 \mathrm{~h} \mathrm{were}$ prepared, treated with RNAse A (10 $\mu \mathrm{g} / \mathrm{ml}$ ) for $30 \mathrm{~min}$, immunoprecipitated with an anti-eEF1A antibody, and tRNALys in the eEF1A/Nef complexes was amplified by RT-PCR. (b) Binding of tRNA to cytochrome $c$ is eEF1A dependent. MDMs were mock treated or treated with BFA $(10 \mu \mathrm{g} / \mathrm{ml})$ or BFA $(10 \mu \mathrm{g} / \mathrm{ml})+\mathrm{Nef}(1000 \mathrm{ng} / \mathrm{ml})$ for $12 \mathrm{~h}$ in the presence of control or eEF1A siRNA. The lysates were immunoprecipitated with anti-cytochrome $c$, and presence and amount of tRNALys were determined by qRT-PCR and further samples were resolved on $3 \%$ agarose gel and stained with $\mathrm{EtBr}$. (c and d) MDMs were treated with recombinant Nef (100 ng/ml); the lysates were immunoprecipitated with an anti-Nef Ab (c) or anti-eEF1A Ab (d) and the presence of tRNAMet, tRNALys, tRNATrp, and tRNAPhe in the Nef/eEF1A complex was determined by qRT-PCR. (e and f) MDMs were treated with BFA for $12 \mathrm{~h}$ in the presence or absence of $\mathrm{rNef}(0-1500 \mathrm{ng} / \mathrm{ml})$; the lysates were immunoprecipitated with an anti-eEF1A Ab (e) or anti-cytochrome $c \mathrm{Ab}$ (f) and the presence of tRNAMet, tRNALys, tRNATrp, and tRNAPhe was determined by qRT-PCR. ( $\mathbf{g}$ and $\mathbf{h})$ The presence of tRNAs associated with eEF1A and cytochrome $c$ in BFA-stimulated MDMs treated with rNef is dependent on Exp-t. MDM cultures were transfected with a scrambled control siRNA, Exp-t siRNA, Exp-1 siRNA, or Exp-5 siRNA. Cytoplasmic extracts of MDMs treated with BFA $(10 \mu \mathrm{g} / \mathrm{ml})$ for $12 \mathrm{~h}$ in the absence or presence of rNef $(1000 \mathrm{ng} / \mathrm{ml})$ were prepared $48 \mathrm{~h}$ post transfection, immunoprecipitated with an anti-eEF1A Ab $(\mathbf{g})$ or anti-cytochrome $c \mathrm{Ab}(\mathbf{h})$, and the presence of tRNALys, tRNAMet, tRNAPhe, and tRNATrp binding to eEF1A and cytochrome $c$ was determined by qRT-PCR. Results representative of three independent experiments are shown. ${ }^{*} P<0.05$. (i) The presence of tRNAs associated with cytochrome $c$ in BFA-stimulated MDMs treated with rNef is dependent on eEF1A. MDM cultures were transfected with control siRNA or eEF1A siRNA. Cytoplasmic extracts of MDMs treated with BFA $(10 \mu \mathrm{g} / \mathrm{ml})$ for $12 \mathrm{~h}$ in the absence or presence of $\mathrm{rNef}(1000 \mathrm{ng} / \mathrm{ml})$ were prepared $48 \mathrm{~h}$ post transfection and were immunoprecipitated with an anti-cytochrome $c$ Ab. The presence of tRNAMet, tRNALys, tRNAPhe, and tRNATrp binding to cytochrome $c$ was determined using qRT-PCR. Results representative of two independent experiments are shown 


\section{Materials and Methods}

Cell culture. Primary human MDMs were prepared from the peripheral blood of healthy donors and cultured in RPMI medium supplemented with $10 \%(\mathrm{v} / \mathrm{v})$ pooled AB human serum (Sigma, Munich, Germany), as described previously. ${ }^{39}$ PBLs were cultivated in RPMI medium supplemented with $10 \%(\mathrm{v} / \mathrm{v})$ fetal bovine serum (FBS). Vero cells, MRC5 cells, and promonocytic U937 cells were obtained from the American Tissue Cell Culture Collection (ATCC, Manassas, VA, USA). The cell lines were cultivated in RPMI 1640 supplemented with 10\% FBS, 1\% glutamine, and $1 \%$ penicillin/streptomycin.

Recombinant Nef treatment. MDMs $\left(5 \times 10^{6}\right.$ cells $)$ were treated with recombinant myristoylated Nef protein (rNef) from Jena Bioscience (Jena, Germany). Cell pellets were collected at various times after treatment, washed extensively, and either lysed for western blot analysis or fixed with $3 \%$ paraformaldehyde in PBS for 30 min for the TUNEL assay in flow cytometric analysis. The bacterially expressed GST-Nef(WT), GST-Nef(1-60) and GSTNef(55-206) were purified and cleaved with thrombin (cat\# 27-0846-01 GE Healthcare Ltd., Little Chalfont, UK) according to the instruction provided by the manufacturer. SDS-tricine polyacrylamide gel electrophoresis (PAGE) and coomassie staining was done to visualize the thrombin cleaved products of HIV-1Nef wild type and mutants.

Isolation of nuclear and cytoplasmic extracts. Nuclear and cytoplasmic extracts were isolated as described previously. ${ }^{39}$ Cells were scraped from the plastic surface of the culture dishes and washed with wash buffer $(10 \mathrm{mM}$ HEPES pH 7.6, $10 \mathrm{mM} \mathrm{KCl}, 2 \mathrm{mM} \mathrm{MgCl} 2,1 \mathrm{mM}$ EDTA). Cell pellets were

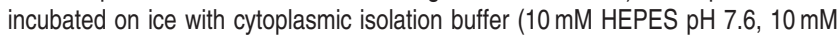
$\mathrm{KCl}, 2 \mathrm{mM} \mathrm{MgCl} 2,1 \mathrm{mM}$ EDTA, $0.02 \%$ Nonidet P-40). Supernatants containing cytoplasmic extracts were collected by centrifugation leaving the nuclear pellets. The pellets were washed twice in wash buffer, spun, and incubated for $15 \mathrm{~min}$ on ice with nuclear isolation buffer (20 mM HEPES pH 7.6, $420 \mathrm{mM} \mathrm{NaCl}, 1.5 \mathrm{mM}$ $\mathrm{MgCl}$, $0.2 \mathrm{mM}$ EDTA, 25\% glycerol). Supernatants containing nuclear extracts were collected by centrifugation and stored at $-80^{\circ} \mathrm{C}$. Protease inhibitors $(1 \mathrm{mM}$ DTT, $1 \mathrm{mM}$ PMSF, $1 \mu \mathrm{g} / \mathrm{ml}$ aprotinin, $1 \mu \mathrm{g} / \mathrm{ml}$ leupeptin, $1 \mu \mathrm{g} / \mathrm{ml}$ pepstatin) were added to all solutions. Protein concentrations in the nuclear and cytoplasmic extracts were determined by the Bradford method using a BioPhotometer (Eppendorf, Hamburg, Germany).

Preparation of cytosolic and mitochondrial fractions. Cytosolic and mitochondrial fractions were prepared using the cell fractionation kit-standard (\#MS861, Mitosciences, Eugene, OR, USA). The coxIV (Cell Signaling Technology, Danvers, MA, USA) antibody was used as a mitochondrial loading control.

Immunoprecipitation. Vero cells, MRC5 cells, U937 cells, PBLs, or primary MDMs were left untreated or treated with rNef for different periods of time. Cell lysates were precleared by adding $50 \mu \mathrm{l}$ of Protein G Plus/Protein A-Agarose (Calbiochem-Novabiochem, Bad Soden, Germany) for $1 \mathrm{~h}$ at $4^{\circ} \mathrm{C}$. The cleared supernatants were removed, combined with $5 \mu \mathrm{g}$ anti-Nef or anti-EF1A antibodies (Chemicon, Upstate, Billerica, MA, USA), and incubated overnight at $4^{\circ} \mathrm{C}$. Immune complexes were washed in the presence of protease inhibitors, the bound proteins eluted with sample buffer, and the proteins run on a 10\% SDS-PAGE gel. SDS-PAGE and western blot analysis were performed according to standard procedures. Beta-actin and TATA-binding protein (TBP) (Sigma, St. Louis, MO, USA) were used as cytoplasmic or nuclear loading controls, respectively. Western blots were developed using the ECL detection kit (Amersham Pharmacia Biotech, Little Chalfont, UK).

Plasmids and construction of expression vectors. Deletion constructs were generated by standard PCR and cloning procedures. ${ }^{40}$ HIV -1 Nef constructs were verified by DNA sequencing. eEF1A/Nef interactions were detected in vivo by transient co-transfection of GAL4-Nef and VP16-eEF1A in the context of a mammalian two-hybrid system (CheckMate, Promega, Madison, WI, USA). Sequence authenticity was confirmed by DNA cycle sequencing. HIV-1 Nef wild type, N-terminus (1-60), or C-terminus (55-206) was cloned into pGEX-4T-1 expression vector. For protein purification purposes, each construct was transformed into bacteria (BL21DE from Novagen, Madison, WI, USA).
A single colony was inoculated in $5 \mathrm{ml} \mathrm{LB}$ broth plus $50 \mu \mathrm{g} / \mathrm{ml}$ ampicillin at $37^{\circ} \mathrm{C}$ in a shaker overnight. A 1-ml aliquot of the overnight bacterial culture was used to inoculate $300 \mathrm{ml} \mathrm{LB}$ broth plus $50 \mu \mathrm{g} / \mathrm{ml}$ ampicillin and grown for $2.5 \mathrm{~h}$ at $37^{\circ} \mathrm{C}$ in a shaker (until $\mathrm{OD} 600=0.5$ ). IPTG was added at a final concentration of $0.1 \mathrm{mM}$ and the culture grown for an additional $3 \mathrm{~h}$ at $37^{\circ} \mathrm{C}$ in the shaker. Bacteria were pelleted, washed with ice-cold TBS, and lysed in bacterial protein extraction reagents (Pierce, Rockford, IL, USA). eEF1A and mutants (kindly provided by Dr. A Cimarelli, ENS-L, Lyon, France) were constructed and expressed in the bacteria, as described previously. ${ }^{24}$

Transfection assay. To carry out transient transfections, the DNA concentration was kept constant in the different samples by using the corresponding empty vector. A total of $5 \times 10^{6}$ cells were transfected with 5-10 $\mu \mathrm{g}$ total plasmid DNA, which included the firefly luciferase-expressing vector using the DEAE-dextran procedure. Luciferase activity in cell lysates was measured $48 \mathrm{~h}$ post transfection using a luminometer (TD-20/20, Promega). Luciferase expression was normalized with respect to protein concentration using the Detergent-Compatible Protein Assay (Bio-Rad, Munich, Germany). All transfections were performed in triplicate.

Pull-down assays. Fragments of the cDNA encoding HIV-189.6 Nef were produced using convenient restriction enzymes and PCR methods and then cloned into the glutathione $S$ transferase (GST) fusion vector pGEX-4T-1 (Amersham Biosciences, Little Chalfont, UK). The GST constructs (Nef[1-60] and Nef[55-206]) kindly provided by Dr. Fackler (University of Heidelberg, Germany) were transformed into the E. coli strain BL21 (Stratagene, La Jolla, CA, USA), and the GST fusion proteins were purified according to the manufacturer's instructions. For pull-down assays, $20 \mu \mathrm{g}$ of the GST-Nef proteins (NefWT, Nef[1-60], Nef[55206]) were incubated overnight at $4^{\circ} \mathrm{C}$ with $1500 \mu \mathrm{g}$ of MDM lysates. The suspension was then washed three times in PBS, denaturized, and subsequently separated by SDS-PAGE before autoradiography.

RNA interference. MDM cultures $\left(0.5 \times 10^{6}\right.$ cells $)$ were transfected with a scrambled control, Exp-t siRNA, Exp-1 siRNA, Exp-5 siRNA duplex (Santa Cruz Biotechnology, Santa Cruz, CA, USA) or eEF1A siRNA (Dharmacon, Lafayette, CO, USA) using Lipofectamine RNAiMAX (Invitrogen, Carlsbad, CA, USA). MDMs were treated with rNef $48 \mathrm{~h}$ post transfection, and eEF1A, rNef, Exp-t, Exp-1, and Exp-5 protein expression and MDM apoptosis were analyzed by western blot and the annexin-V (Invitrogen) assay, respectively. Transfection efficiency was monitored using a fluorescein-conjugated scrambled control duplex, exceeding $80 \%$ in MDMs.

tRNA interaction with cytochrome $\boldsymbol{c}$, eEF1A, or Nef. To examine the tRNA/cytochrome $c$ interaction in vivo, MDMs transfected with Exp-t siRNA, Exp-1 siRNA, Exp-5 siRNA, eEF1A siRNA or scramble siRNA for $48 \mathrm{~h}$ before treatment with rNef were harvested and washed twice with ice-cold PBS. The cytoplasmic extracts were immunoprecipitated with anti-cytochrome $c$ antibody (Santa Cruz Biotechnology) at $4{ }^{\circ} \mathrm{C}$ overnight followed by incubation with protein $\mathrm{G}$ agarose beads for an additional $2 \mathrm{~h}$. The beads were washed and resuspended in $200 \mu$ l RNA elusion buffer ( $50 \mathrm{mM}$ Tris, $100 \mathrm{mM} \mathrm{NaCl}, 10 \mathrm{mM}$ EDTA, 0.1\% SDS) at $65^{\circ} \mathrm{C}$ for $10 \mathrm{~min}$. RNA was extracted (RNeasy kit, Qiagen, Valencia, CA, USA) and cDNA synthesized using a cDNA synthesis kit (Invitrogen).

The sequence of the primers (Eurogentec, Seraing, Belgium) used in RT-PCR amplification were as follows:

tRNAMet forward 5'-CTGGGCCCATAACCCAGAG-3'

tRNAMet reverse 5'-TAGCAGAGGATGGTTTCGAT-3'

tRNATrp forward 5'-GGCTCGTTGGTCTAGGGGTA-3'

tRNATrp reverse 5'-GATTTGAACCCGGGACCT-3'

tRNAPhe forward $5^{\prime}$-CCTCCTCAAAGCAATACACTGA-3'

tRNAPhe reverse $5^{\prime}$-GGTGATGTGAGCCCGTCTAA-3'

tRNALys forward 5'-ATAGCTCAGTCGGTAGAGCATCA-3'

tRNALys reverse 5'-ACAGGGACTTGAACCCTGGAC-3'

The tRNAs associated with eEF1A, rNef and cytochrome $c$ were detected after immunoprecipitation with an anti-eEF1A and anti-cytochrome $c$ or anti-Nef antibody, followed by RT-PCR amplification as described above. Ct values were analyzed using the $\Delta \Delta \mathrm{Ct}$ method. ${ }^{41} \Delta \mathrm{Ct}$ was calculated by normalization to the isotype control, and $\Delta \Delta \mathrm{Ct}$ values were calculated by normalizing to the $\mathrm{Ct}$ values of the untreated sample. The $\Delta \Delta \mathrm{Ct}$ values were also determined separately for untreated cells and averaged around 1 (Supplementary Figure 2). 
Detection of apoptosis. The detection of apoptosis by the annexinV-propidium iodide assay (Invitrogen) and TUNEL assay (AbD Serotec, Oxford, UK was performed as previously described ${ }^{28}$ For ER stress agents, an appropriate dose was selected from a range of concentrations tested. BFA (Sigma), TG (Sigma) and TM (Sigma) were used at $10 \mu \mathrm{g} / \mathrm{ml}$.

Caspase activity. Caspase- 9 and caspase- 3 activity was measured by western blotting as described previously. ${ }^{30}$ Where specified, protein levels were quantified by densitometry using ImageJ 1.40 software (National Institutes of Health, Bethesda, MA, USA).

Statistical analysis. Statistical analyses were performed using the analysis of variance (ANOVA) test, and differences were considered significant at a value of ${ }^{*} P<0.05,{ }^{* *} P<0.01,{ }^{* *} P<0.001$. Microsoft Excel was used to construct the plots.

\section{Conflict of Interest}

The authors declare no conflict of interest.

Acknowledgements. This work was supported by grants from the University of Franche-Comté (UFC) to GH. OR is supported by grants from University of Strasbourg. Agence Nationale de la Recherche sur le SIDA (ANRS), Ligue Nationale de Recherche contre le Cancer and Institut Universitaire de France (IUF). WA and KAK are recipients of doctoral scholarships from the Higher Education Commission, Pakistan. AK is a recipient of a postdoctoral fellowship of the Région Franche-Comté (no. 2012C-06102).

1. Walter $P$, Ron $D$. The unfolded protein response: from stress pathway to homeostatic regulation. Science 2011; 334: 1081-1086.

2. Shore GC, Papa FR, Oakes SA. Signaling cell death from the endoplasmic reticulum stress response. Curr Opin Cell Biol 2011; 23: 143-149.

3. Elbim C, Pillet S, Prevost MH, Preira A, Girard PM, Rogine N et al. Redox and activation status of monocytes from human immunodeficiency virus-infected patients: relationship with viral load. J Virol 1999; 73: 4561-4566.

4. Masutani $\mathrm{H}$, Ueda S, Yodoi J. The thioredoxin system in retroviral infection and apoptosis. Cell Death Diff 2005; 12: 991-998.

5. Uhm HD, Orenstein JM, Wahl SM. Fas mediates apoptosis in human monocytes by a reactive oxygen intermediate dependent pathway. J Immunol 1996; 156: 3469-3477.

6. Aillet $\mathrm{F}$, Masutani $\mathrm{H}$, Elbim $\mathrm{C}$, Raoul $\mathrm{H}$, Chene L, Nugeyre MT et al. Human immunodeficiency virus induces a dual regulation of $\mathrm{Bcl}-2$, resulting in persistent infection of CD4 + T- or monocytic cell lines. J Virol 1998; 72: 9698-9705.

7. Tiedde LM, Cook EA, Morsey B, Fox HS. Oxygen matters: tissue culture oxygen levels affect mitochondrial function and structure as well as responses to HIV viroproteins. Cell Death Dis 2011; 2: e246.

8. Kohler A, Hurt E. Exporting RNA from the nucleus to the cytoplasm. Nat Rev Mol Cell Biol 2007; 8: 761-773.

9. Calado A, Treichel N, Muller EC, Otto A, Kutay U. Exportin-5-mediated nuclear export o eukaryotic elongation factor $1 \mathrm{~A}$ and tRNA. EMBO J 2002; 21: 6216-6224.

10. Liu G, Grant WM, Persky D, Latham VM Jr, Singer RH, Condeelis J. Interactions of elongation factor 1 alpha with F-actin and beta-actin mRNA: implications for anchoring mRNA in cell protrusions. Mol Biol Cell 2002; 13: 579-592.

11. Amiri A, Noei F, Jeganathan S, Kulkarni G, Pinke DE, Lee JM. eEF1A2 activates Akt and stimulates Akt-dependent actin remodeling, invasion and migration. Oncogene 2007; 26 3027-3040.

12. Murray JW, Edmonds BT, Liu G, Condeelis J. Bundling of actin filaments by elongation factor 1 alpha inhibits polymerization at filament ends. J Cell Biol 1996; 135 : 1309-1321.

13. Duttaroy A, Bourbeau D, Wang XL, Wang E. Apoptosis rate can be accelerated or decelerated by overexpression or reduction of the level of elongation factor-1 alpha. Exp Cell Res 1998; 238: 168-176.

14. Lamberti A, Longo O, Marra M, Tagliaferri P, Bismuto E, Fiengo A et al. C-Raf antagonizes apoptosis induced by IFN-alpha in human lung cancer cells by phosphorylation and increase of the intracellular content of elongation factor 1A. Cell Death Differ 2007; 14: 952-962.
15. Mateyak MK, Kinzy TG. eEF1A: thinking outside the ribosome. J Biol Chem 2010; 285: 21209-21213

16. Foster JL, Garcia JV. HIV-1 Nef: at the crossroads. Retrovirology 2008; 5: 84

17. Ross TM, Oran AE, Cullen BR. Inhibition of HIV-1 progeny virion release by cell-surface CD4 is relieved by expression of the viral Nef protein. Curr Biol 1999; 9: 613-621.

18. Kirchhoff $F$. Immune evasion and counteraction of restriction factors by HIV-1 and other primate lentiviruses. Cell Host Microbe 2010; 8: 55-67.

19. Collins KL, Chen BK, Kalams SA, Walker BD, Baltimore D. HIV-1 Nef protein protects infected primary cells against killing by cytotoxic T lymphocytes. Nature 1998; 391: 397-401.

20. Geleziunas R, Xu W, Takeda K, Ichijo H, Greene WC. HIV-1 Nef inhibits ASK1- dependent death signalling providing a potential mechanism for protecting the infected host cell. Nature 2001; 410: 834-838.

21. Wolf D, Witte V, Laffert B, Blume K, Stromer E, Trapp S et al. HIV-1 Nef associated PAK and $\mathrm{PI} 3$-kinases stimulate Akt-independent Bad-phosphorylation to induce anti-apoptotic signals. Nat Med 2001; 7: 1217-1224

22. Olivetta E, Federico M. HIV-1 Nef protects human-monocyte-derived macrophages from HIV-1-induced apoptosis. Exp Cell Res 2006; 312: 890-900.

23. Herbein G, Gras G, Khan KA, Abbas W. Macrophage signaling in HIV-1 infection. Retrovirology 2010; $7: 34$

24. Cimarelli A, Luban J. Translation elongation factor 1-alpha interacts specifically with the human immunodeficiency virus type 1 Gag polyprotein. J Virol 1999; 73: 5388-5401.

25. Alessandrini L, Santarcangelo AC, Olivetta E, Ferrantelli F, d'Aloja P, Pugliese K et al. T-tropic human immunodeficiency virus (HIV) type 1 Nef protein enters human monocytemacrophages and induces resistance to HIV replication: a possible mechanism of HIV T-tropic emergence in AIDS. J Gen Virol 2000; 81: 2905-2917.

26. Li J, Yuan J. Caspases in apoptosis and beyond. Oncogene 2008; 27: 6194-6206.

27. Guo H, Tittle TV, Allen H, Maziarz RT. Brefeldin A-mediated apoptosis requires the activation of caspases and is inhibited by Bcl-2. Exp Cell Res 1998; 245: 57-68.

28. Talapatra S, Wagner JD, Thompson CB. Elongation factor-1 alpha is a selective regulator of growth factor withdrawal and ER stress-induced apoptosis. Cell Death Diff 2002; 9: 856-861

29. Kobayashi $\mathrm{Y}$, Yonehara S. Novel cell death by downregulation of eEF1A1 expression in tetraploids. Cell Death Diff 2009; 16: 139-150.

30. Mei Y, Yong J, Liu H, Shi Y, Meinkoth J, Dreyfuss $\mathrm{G}$ et al. tRNA binds to cytochrome $\mathrm{c}$ and inhibits caspase activation. Mol Cell 2010; 37: 668-678.

31. Echarri A, Gonzalez ME, Carrasco L. The N-terminal Arg-rich region of human immunodeficiency virus types 1 and 2 and simian immunodeficiency virus Nef is involved in RNA binding. Eur J Biochem 1997; 246: 38-44.

32. Nissen P, Kjeldgaard M, Thirup S, Polekhina G, Reshetnikova L, Clark BF et al. Crystal structure of the ternary complex of Phe-tRNAPhe, EF-Tu, and a GTP analog. Science 1995: 270: 1464-1472.

33. Fackler OT, Baur AS. Live and let die: Nef functions beyond HIV replication. Immunity 2002; 16: 493-497.

34. Chuang SM, Chen L, Lambertson D, Anand M, Kinzy TG, Madura K. Proteasomemediated degradation of cotranslationally damaged proteins involves translation elongation factor $1 \mathrm{~A}$. Mol Cell Biol 2005; 25: 403-413.

35. Hotokezaka Y, Tobben U, Hotokezaka H, Van Leyen K, Beatrix B, Smith DH et al. Interaction of the eukaryotic elongation factor $1 \mathrm{~A}$ with newly synthesized polypeptides. J Biol Chem 2002; 277: 18545-18551.

36. Nicchitta CV. Cell biology: how to combat stress. Nature 2009; 457: 668-669.

37. Resh MD. Use of analogs and inhibitors to study the functional significance of protein palmitoylation. Methods 2006; 40: 191-197.

38. Michelangeli F, East JM. A diversity of SERCA Ca2 + pump inhibitors. Biochem Soc Trans 2011; 39: 789-797.

39. Varin A, Manna SK, Quivy V, Decrion AZ, Van Lint C, Herbein G et al. Exogenous Nef protein activates NF-kappa B, AP-1, and C-Jun N-terminal kinase and stimulates HIV transcription in promonocytic cells. Role in AIDS pathogenesis. J Biol Chem 2003; 278: 2219-2227.

40. Emiliani S, Fischle W, Van Lint C, Al-Abed Y, Verdin E. Characterization of a human RPD3 ortholog, HDAC3. Proc Natl Acad Sci USA 1998; 95: 2795-2800.

41. Livak KJ, Schmittgen TD. Analysis of relative gene expression data using real-time quantitative PCR and the 2( - Delta Delta C(T)) Method. Methods 2001; 25: 402-408.

(1) (1) Cell Death and Disease is an open-access journa published by Nature Publishing Group. This work is licensed under a Creative Commons Attribution-NonCommercialShareAlike 3.0 Unported License. To view a copy of this license, visit http://creativecommons.org/licenses/by-nc-sa/3.0/ 